\title{
Installation of Stiffened Caissons in Non-Homogeneous Clays
}

\section{Mi Zhou ${ }^{1}$, Muhammad Shazzad Hossain ${ }^{2}$, Yuxia $\mathrm{Hu}^{3}$ and Hanlong Liu ${ }^{4}$}

\begin{abstract}
Significant difference between predicted and measured installation resistance of stiffened suction caissons was identified due to the existing uncertainty regarding the mobilized soil flow mechanisms. This paper describes an extensive investigation of stiffened caisson penetration in non-homogeneous clays undertaken through large deformation finite element (LDFE) analysis to provide insight into the soil behavior during installation of a caisson. The soil flow mechanisms around and between stiffeners, and inside and outside of the caisson, and the corresponding penetration resistances were presented from a parametric study, exploring a range of dimensionless parameters related to stiffened caisson geometry, caisson roughness and soil strength non-homogeneity.
\end{abstract}

The LDFE results were compared with centrifuge test data in terms of the soil flow mechanisms and penetration resistance profile, with good agreement obtained. Three interesting features in the mobilized soil flow mechanisms inside the caisson were

\footnotetext{
${ }^{1}$ Research Associate (PhD), Hohai University, 1 Xikang Rd, Nanjing, PR China, 210098; School of Civil, Environmental and Mining Engineering, The University of Western Australia, 35 Stirling Highway, Crawley, WA 6009, Tel: +61 86488 3071, Fax: +61 86488 1018, Email: mi.zhou@uwa.edu.au

${ }^{2}$ Corresponding Author, Associate Professor (BEng, MEng, PhD, MIEAust), ARC DECRA Fellow, Centre for Offshore Foundation Systems (COFS), The University of Western Australia, 35 Stirling Highway, Crawley, WA 6009, Tel: +61 86488 7358, Fax: +61 86488 1044, Email: muhammad.hossain@uwa.edu.au

${ }^{3}$ Professor (PhD, MIEAust), School of Civil, Environmental and Mining Engineering, The University of Western Australia, 35 Stirling Highway, Crawley, WA 6009, Tel: +61 86488 8182, Fax: +61 86488 1018, Email: yuxia.hu@uwa.edu.au

${ }^{4}$ Professor (PhD), College of Civil and Transportation Engineering, Key Laboratory of Ministry of Education for Geomechanics and Embankment Engineering, Hohai University, Nanjing 210098, China, Tel: +86 258378 6918, Fax: +86 258371 3073, E-mail: hliuhhu@163.com
} 
observed: (i) soil started to infill the gap between the bottom two stiffeners when the bottom stiffener penetration reached $H_{r}$; (ii) soil started to infill the gap between the second and third bottom stiffeners when the second stiffener penetration reached $H_{c}$; (iii) soil heaving inside the caisson. Both $H_{\mathrm{r}}$ and $H_{\mathrm{c}}$ were shown to be a function of stiffener width ratio to the caisson diameter, normalized soil strength at the mudline and soil strength non-homogeneity. To predict the penetration resistance profile in the field, a rational analytical penetration model, based on the revealed soil flow mechanisms, was proposed, with the LDFE data were used to calibrate the model. Very good agreements were obtained when the proposed model was validated against measured data from field installation and centrifuge tests.

CE Database subject headings: Clays; Caissons; Flow patterns; Numerical analysis; Offshore structures; Penetration resistance; Stiffeners. 


\section{Notation}

$A_{\mathrm{b}} \quad$ area of bottom stiffener base

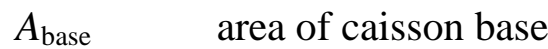

$A_{\mathrm{i}} \quad$ Inner wall area of skirt below mudline

$A_{\text {iab }} \quad$ Inner area above bottom stiffener and below limiting cavity depth

$A_{\mathrm{ib}} \quad$ Inner wall area of embedded skirt below bottom stiffener

$A_{0} \quad$ outer wall area of skirt below mudline

$A_{\mathrm{t}} \quad$ area of skirt tip

$b \quad$ width of stiffener

D diameter of caisson

$d \quad$ penetration depth of skirt tip

$d_{\mathrm{b}} \quad$ penetration depth of bottom stiffener

$d_{2} \quad$ penetration depth of $2^{\text {nd }}$ stiffener

F total penetration resistance of caisson

$F_{\mathrm{i}} \quad$ inner frictional resistance

$F_{\mathrm{ib}} \quad$ inner frictional resistance below bottom stiffener

$F_{\text {iabb }} \quad$ inner frictional resistance along faces above bottom stiffeners

$F_{0} \quad$ outer skirt wall frictional resistance

$F_{\mathrm{t}} \quad$ end bearing at skirt tip 


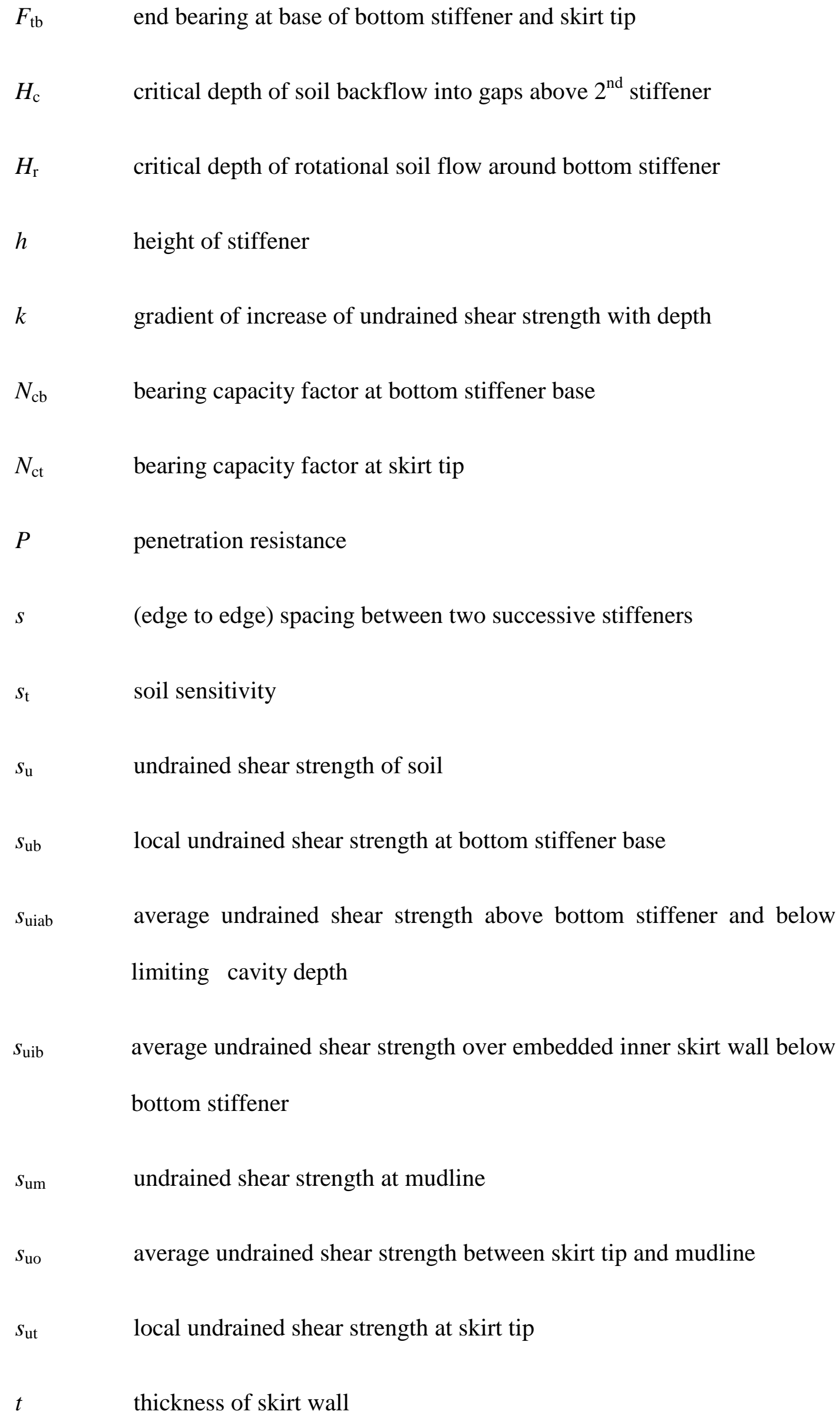


distance of bottom stiffener base from skirt tip

$Z$

depth below soil surface

$\alpha$

coefficient of interface friction

$\gamma^{\prime}$

effective unit weight of soil

$\chi$

coefficient of inner friction resistance above bottom stiffener 


\section{Introduction}

\section{Stiffened Suction Caisson and Corresponding Design Uncertainties}

Suction caissons are thin-walled (thickness, $t \leq 50 \mathrm{~mm}$; see schematic in Figure 1a) and thus prone to buckling during installation or distortion under the anchoring load. As such, horizontal ring stiffeners at intervals along the inner wall of the thin skirt are employed with local thickening of the wall in the vicinity of the padeye, with or without transverse struts.

The addition of these stiffeners has created significant uncertainties regarding the soil flow mechanisms, skirt wall friction and end bearing, and consequently in the prediction of underpressure required for installation. In absence of any relevant investigation, previously, three soil flow mechanisms were assumed (see Figure 2) for assessing stiffened caisson penetration response in the field. However, the predicted underpressures based on these mechanisms were significantly higher than the measured ones (see Figure 3; Dendani and Colliat 2002; Erbrich and Hefer 2002; Dendani 2003). In exercise calculations, significant variations among the predictors for various cases were also identified by Andersen (Andersen et al. 2005). This was largely due to the assumptions of different soil flow mechanisms around and between the embedded stiffeners.

Some consequent uncertainties in predicting the caisson installation resistance include: (a) the length of caisson walls contributing to frictional resistance; (b) the end bearing at the base of the stiffeners above the bottom one; (c) the operational shear strength (remoulded, residual or intact) and (d) the interface friction coefficient, $\alpha$. In essence, an improved understanding of the soil flow mechanisms between the stiffeners during 
caisson installation is essential for accurate prediction of the underpressure required to achieve targeted penetration depth.

\section{Previous Work}

Unstiffened caisson installation in clay has been addressed through large deformation finite element (LDFE) analyses and centrifuge model tests by House and Randolph (2001), Randolph and House (2002), Zhou and Randolph (2006), Chen and Randolph (2007), Chen et al. (2009), Westgate et al. (2009), Vásquez et al. (2010) among others. They noticed no discernible difference in penetration resistance between jacked and suction-installed caisson, except Zhou and Randolph (2006). Significant proportion of soil flow was reported to occur inward into the caisson, with little outward flow.

Installation of caissons with stiffeners has not attracted much research until recently, although mostly through centrifuge tests (House and Randolph 2001; Randolph et al. 1998; Westgate et al. 2009). The addition of the horizontal and vertical stiffeners was reported to have increased the required installation pressure significantly due to a combination of additional stiffener end bearing and stiffener side friction, offset by reductions of internal skirt side friction (Westgate et al. 2009). Hossain et al. (2012) carried out centrifuge tests on skirt plates with stiffeners penetrating into uniform and non-homogeneous clays (as discussed later).

\section{Objective of Present Study}

This paper reports the results from an extensive investigation carried out through LDFE analysis in an attempt to provide the 'ground truth' of stiffened caisson penetration mechanisms in non-homogeneous clays. An extensive parametric investigation was undertaken, varying the relevant range of various parameters related to the caisson geometry and soil strength. A new penetration mechanism and, based 
on that, a rational analytical model are then proposed for assessing the underpressure required for installation of stiffened caissons in the field.

\section{Large Deformation FE Analysis}

This study has considered a stiffened caisson of diameter $D$ and length $L$ penetrating into a non-homogeneous clay deposit as illustrated schematically in Figure 1, where the undrained shear strength of clay, $s_{\mathrm{u}}$, increases linearly with depth from a mudline intercept of $s_{\text {um }}$ with a gradient of $k$. The thickness of the caisson skirt is $t$. The ring stiffeners of width $b$, height $h$, and with edge to edge spacing s, are placed along the inner wall of the skirt, with the bottom stiffener positioned at a distance of $w$ from the skirt tip.

Large deformation finite element (LDFE) analyses were undertaken using RITSS (Remeshing and Interpolation Technique with Small Strain; Hu and Randolph 1998) method implemented in the finite element package AFENA (Carter and Balaam 1995), which was developed at the University of Sydney. The RITSS method falls within what are known as arbitrary Lagrangian-Eulerian (ALE) finite element methods (Ghosh and Kikuchi 1991), whereby a series of small strain analysis (using AFENA) are combined with frequent fully automatic remeshing of the entire domain, followed by interpolation of all field variables (such as stresses and material properties) from the old mesh to the new mesh.

Six-noded triangular elements with three internal Gauss points were used in all the FE analyses. The skirt- and stiffeners-soil interfaces were simulated using elastoplastic nodal joint elements (Herrmann 1978) distributed along the caisson-soil contact. The shear strength of the nodal joint elements was limited to $\alpha s_{\mathrm{u}}$, where $\alpha$ is the interface 
friction coefficient and $s_{\mathrm{u}}$ is the local undrained shear strength of the soil. The simulation of penetration was achieved by specifying downward displacement increment of the caisson represented by a rigid skirt.

In order to isolate each resistance component at each specific boundary (e.g. at the tip of the skirt, along the outer embedded surface of the skirt), the existing code was extended to record and track the resistance forces on separate boundaries before and after each remeshing.

The axisymmetric soil domain was chosen as $10 D$ in radius and $10 D$ in depth to ensure that the domain boundaries were well outside the plastic zone. Hinge and roller conditions were applied along the base and vertical sides of the soil domain respectively. The soil was modelled as an elastoplastic material obeying a Tresca yield criterion, with an associated flow rule, apart from rounding of the corners of the hexagon (Sloan and Booker 1986). All the analyses were conducted based on total stress, which simulated undrained soil conditions and adopted a Poisson's ratio $v=0.49$ (sufficiently high to give minimal volumetric strains, while maintaining numerical stability), friction and dilation angles $\phi=\psi=0$, and a uniform stiffness ratio $E / s_{\mathrm{u}}=500$ (where $E$ is the Young's modulus) throughout the soil domain. The stiffness ratio is within the range commonly adopted for soft clays. Typical computation times on a fast desktop computer were about 24 hours for a caisson tip penetration of 100t. The geostatic stress conditions were modelled using $K_{0}=1$, as the ultimate penetration resistance was found to be unaffected by the value of $K_{0}$ (Zhou and Randolph 2009).

Strain softening was not considered in the soil constitutive model implemented. The interface friction coefficient $\alpha$ is believed to provide good indication of its effect. 
Furthermore, for quasi-static installation of caissons, the rate dependency of shear strength and strain softening will have negligible influence on the caisson behaviour, as mapped closure to triaxial test by Randolph et al. (2007). This effect of softening has not been modelled in previous relevant studies (Zhou and Randolph 2006; Chen et al. 2009; Westgate et al. 2009; Vásquez et al. 2010).

\section{Results and Discussion}

\section{Validation against Centrifuge Test Data}

The results from LDFE analyses were validated against the observed soil failure mechanisms and measured penetration resistance from centrifuge tests. To visualize the soil flow mechanisms, Hossain et al. (2012) performed centrifuge tests on two skirt plates, penetrating adjacent to the strongbox window. The displacement vectors were quantified through particle image velocimetry (PIV) analysis. The test was carried out at $100 \mathrm{~g}$ on non-homogeneous clay with $s_{\mathrm{u}}=10+0.6 \mathrm{z} \mathrm{kPa}$. Horizontal stiffeners were included on both sides of the plate skirt $(s / h=2, b / t=2$ and $4, w / b=$ 5.33 and 2.67, $S_{\mathrm{t}}=2.5 \sim 3$ ). Figure 4 shows a comparison between the model test and the LDFE simulation (using these parameters and adopting $\alpha=0.3$; Group I, Table 1). Good agreement can be seen in terms of key features of the flow mechanisms: surface heave, formation of gaps above and between the embedded stiffeners, pattern and amount of infill soil into the gap between stiffeners.

Gaudin et al. (2014) reported a centrifuge test data for a stiffened caisson of diameter $8 \mathrm{~m}$ installing in high plasticity Gulf of Guinea clay with $s_{\mathrm{u}}=1.33+1.2 \mathrm{z} \mathrm{kPa}\left(\mathrm{kD} / \mathrm{s}_{\mathrm{um}}\right.$ $\left.=7.22, s_{\mathrm{um}} / \gamma^{\prime} D=0.03, S_{\mathrm{t}} \sim 1.8\right)$. A total of 5 internal ring stiffeners were included along the inner wall of the skirt $(D / t=100, b / t=7.3, s / h=19, w / b=6.9)$. An LDFE simulation was performed using these data and $\alpha=0.3$ (as suggested by Gaudin et al. 
2014; Group II, Table 1). The measured and computed profiles are consistent, as shown in Figure 5a.

Comparison was also made with the centrifuge test data reported by Westgate et al. (2009). A stiffened caisson of diameter $11.3 \mathrm{~m}$ was jacked in kaolin clay with $s_{\mathrm{u}}=10$ $+2.8 z \mathrm{kPa}\left(k D / s_{\mathrm{um}}=3.16, s_{\mathrm{um}} / \gamma^{\prime} D=0.15, S_{\mathrm{t}} \approx \sim 3, \mathrm{~s} / \mathrm{h}=3, b / t=3.4, w / b=5.15\right)$. Again, the result from the corresponding LDFE analysis (using $\alpha=0.3$ ) shows an excellent agreement with the measured data (see Figure 5b).

These validation analyses have confirmed the suitability of the LDFE/RITSS method in capturing the soil flow mechanisms and assessing the penetration resistance during stiffened caisson penetration in non-homogeneous clay. Moreover, the used clays in centrifuge tests have sensitivity $S_{\mathrm{t}}>1$, with the LDFE results without soil strain softening model confirming that a suitable friction coefficient $\alpha$ can take care of its effect. The clay used in Gaudin et al. (2014) has low sensitivity $\left(S_{\mathrm{t}} \sim 1.8\right)$, but high degree of non-homogeneity $\left(k D / s_{\mathrm{um}}=7.22\right)$; and the clay used by Westgate et al. (2009) has relatively high sensitivity $\left(S_{\mathrm{t}} \sim 3\right)$, but low degree of non-homogeneity $\left(k D / s_{\text {um }}=3.16\right)$. The complimentary effects from $S_{\mathrm{t}}$ and $k D / s_{\text {um }}$ made the LDFE results with $\alpha=0.3$ fit both centrifuge test data. This phenomenon will be observed in the parametric study. Vásquez et al. (2010) also obtained good overall agreement between computed penetration resistance and measured data without incorporating strain softening in the implemented constitutive model. The results from the parametric study, as assembled in Table 1, are discussed in detail below.

\section{Soil Flow Mechanisms: Effect of Various Factors}

Parametric analyses were performed varying (i) the ratio of the caisson diameter to the thickness of the skirt, $D / t$; (ii) the ratio of stiffener width to the thickness of the 
skirt, $b / t$; (iii) the normalized clay strength, $s_{\mathrm{um}} / \gamma^{\prime} D$, and corresponding nonhomogeneity factor, $k D / s_{\text {um }}$; (iv) the ratio of stiffener spacing to its height, $s / h$; (v) the coefficient of soil-skirt interface friction, $\alpha$.

\section{Evolution of Soil Flow Patterns}

In general, as a caisson penetrates into soft non-homogeneous clay, four interesting features of soil flow can be identified: (i) during initial penetration, the bearing failure occurs at the skirt tip and the bottom stiffener base, with the soil around the bottom stiffener extending upward to the soil surface; (ii) the soil heave is more profound inside the caisson due to significant inward flow and the presence of stiffeners and gap between stiffeners; (iii) the gaps between the embedded stiffeners are filled with water trapped by the low permeability clay; (iv) with the progress of penetration, a rotational flow mechanism around the edge of the bottom stiffener appears and the $2^{\text {nd }}$ stiffener scrapes the deformed soil wall into the gap (e.g. see Figure 6a; Group III, Table 1). The depth of the bottom stiffener base at this penetration is identified as the critical depth of rotational soil flow around the bottom stiffener $H_{\mathrm{r}}$. Subsequently, the bottom gap is filled gradually with further penetration of the caisson.

The infilling process for the gaps above the bottom one occurs later than that for the bottom gap. This is related to the penetration depth of the lower stiffener of the gap. Thus, for any other stiffeners above the bottom one, when its base penetration depth reaches the critical depth, $H_{\mathrm{c}}$ (referred to as limiting cavity depth), soil infilling starts to occur into the gap above it (e.g. see Figure 6b).

It is found that three factors: $D / t ; b / t$ and $k D / s_{\mathrm{um}}, s_{\mathrm{um}} / \gamma^{\prime} D$ influenced the failure mechanisms including critical depths of soil flow, $H_{\mathrm{c}}$ and $H_{\mathrm{r}}$, significantly, as discussed below. 


\section{Effect of Caisson Diameter (D/t)}

To examine the influence of $D / t$ on the soil failure mechanisms, analyses have been carried out by varying caisson diameter, giving the ratio $D / t=90$ and 240 , and soil strength non-homogeneity and normalized strength, $k D / s_{\mathrm{um}}=0.68$ and $1.8, s_{\mathrm{um}} / \gamma^{\prime} D=$ 0.37 and 0.14 respectively, but with identical $b / t=3.4, s / h=4(w / b=5.88, \alpha=0.2$; Group IV, Table 1). Figures 7a and 7b show the soil flow patterns at a penetration of $d / t=90(d / D=1$ and 0.38 respectively). Interestingly, for a very low $D / t$ of 90 (Figure 7a), the soil does not tend to flow back into the gaps between the embedded stiffeners, instead flows vertically upward to the surface. This is because that, at the current penetration depth $(d / t=90)$, the corresponding bottom stiffener penetration $d_{\mathrm{b}} / D=(d-$ $w) / D=0.78$ is lower than $H_{\mathrm{r}} / D=0.94$ and the bottom second stiffener penetration depth $d_{2} / D=(d-w-h-s) / D=0.5$ is much lower than $H_{\mathrm{c}} / D=1.07$. For a higher ratio of $D / t=240$ (Figure 7b), the rotational soil flow around the bottom stiffener starts to occur at $d / t=72.8$ (i.e. $H_{\mathrm{r}} / D=0.22$ ). Thus, the bottom gap is nearly filled completely. This attributes the fact of the lower $D / t$ yielding lower lateral pressure or delayed soil backflow into the gaps, resulting in more upward flow. This is confirmed by the double soil heave height inside the caisson for $D / t=90$ (note, $s_{\mathrm{um}} / \gamma^{\prime} D$ was also significantly higher, 0.37 compared to 0.14 ). This phenomenon is commonly identified for open-ended driven piles (API, 2007). Even for $D / t=240$, no backflow occurred in the other cavities as the current penetration gives $d_{2} / D=0.19<H_{\mathrm{c}} / D=$ 0.35 .

\section{Effect of Stiffener Width and Coefficient of Friction $(b / t$ and $\alpha)$}

In order to show the effect of the relative width of stiffeners, soil failure patterns are displayed in Figures 8a and b for b/t of 3.4, 7, but with identical caisson diameter ratio 
$D / t=160$ and normalized clay strength of $s_{\mathrm{um}} / \gamma^{\prime} D=0.21(s / h=4, b / t=3.4,7, \alpha=0.2$, $k D / s_{\mathrm{um}}=0.8$; Group IV, Table 1). At a penetration of $d / t=102$ (four stiffeners are below the soil surface), soil backflow occurs only in the bottom gap (e.g. for Figure 8a, $d_{\mathrm{b}} / D=0.51>H_{\mathrm{r}} / D=0.3$ and $\left.d_{2} / D=0.36<H_{\mathrm{c}} / D=0.45\right)$. The infill portion of the bottom gap decreases with increasing stiffener width as about $90 \%$ and $30 \%$ for $b / t=$ 3.4 and 7, respectively. The wider the ring stiffener is, the greater the gap volume, requiring more effort to fill it.

In order to show the effect of coefficient of friction, caisson geometry and soil profile were kept identical to Figures 8a and 8b, and the coefficient of friction $\alpha$ varied from 0 to 0.4 . Very similar observations to Figures $8 \mathrm{a}$ and $\mathrm{b}$ with $\alpha=0.2$ were obtained, hence the flow mechanisms are not presented here. This confirms that coefficient of friction (for the range studied) has minimal effect on the soil flow mechanisms.

Moreover, the height of the soil heave inside the caisson increases significantly with increasing width of stiffeners due to the larger volume of stiffeners and gaps. For instance, for $b / t=3.4$ in Figure 8a, the surface is heaved by $0.5 \mathrm{~m}$; which increased to $0.9 \mathrm{~m}$ for $b / t=7$ (see Figure $8 \mathrm{~b}$ ). These effects of normalized stiffener width are consistent with the observations from centrifuge tests (Hossain et al. 2012)

\section{Effect of Soil Strength $\left(k D / s_{\mathrm{um}}, s_{\mathrm{um}} / \gamma^{\prime} \mathrm{D}\right)$}

Two cases with different soil strength profiles were chosen (Groups III and VI, Table 1) to examine the effect of the soil strength non-homogeneity and the normalized strength on the installation mechanisms. Figure 9 shows the patterns for $k D / s_{\mathrm{um}}=22.6$ and $s_{\mathrm{um}} / \gamma^{\prime} D=0.0074(D / t=226, b / t=3.4, s / h=4, w / b=5.15, \alpha=0.2$; Group VI, Table 1). In this very soft deposit with nearly zero strength at the mudline (high $k D / s_{\text {um }}$ and low $s_{\text {um }} / \gamma^{\prime} D$ ), soil backflow above the bottom stiffener occurs very shortly 
prior to penetration of the $2^{\text {nd }}$ stiffener into the ground (see Figure 9a). In addition, the soil strength is insufficient to maintain a vertical wall between the bottom and $2^{\text {nd }}$ stiffeners, instead it collapses gradually into the gap. This type of infilling process occurs to all the gaps between the embedded stiffeners (see Figure 9b). The initial surface heave has caved in completely. In this very soft normally consolidated clay, two critical depths are found to be similar $\left(H_{r} \approx H_{\mathrm{c}} \approx 0.4 \mathrm{~m}\right)$.

The failure patterns for a clay with lower $k D / s_{\mathrm{um}}=2.26$ and higher $s_{\mathrm{um}} / \gamma^{\prime} D=0.074$, but with identical other parameters, are shown previously in Figure 6 (Group III, Table 1). The soil backflow around the bottom stiffener occurs at a greater depth (see Figures $6 a$ and 9a). The higher $s_{\text {um }}$ allowed the soil wall to stand vertically between the stiffeners, leading to no wall collapse. For this case, $H_{\mathrm{r}} / D=0.15$ and $H_{\mathrm{c}} / D=$ 0.18 .

\section{Depth of Rotational Flow $\left(H_{r}\right)$ and Limiting Cavity Depth $\left(H_{c}\right)$}

From the observations of soil failure patterns in Figures 6 9, it can be seen that, for a constant soil effective unit weight, the critical depths $H_{\mathrm{r}}$ and $H_{\mathrm{c}}$ are influenced by the soil strength at the mudline $\left(s_{\mathrm{um}}\right)$, strength gradient with depth $(k)$, caisson diameter (D) and marginally by the stiffener width $(b)$. In order to establish a design chart, an extensive parametric study was undertaken by LDFE/RITSS analysis, varying the principal parameters as detailed below (Group VII, Table 1):

Caisson diameter, $D=4.5,6,8,11.3$ and $15 \mathrm{~m}$;

Mudline strength, $s_{\mathrm{um}}=0.5,5$ and $10 \mathrm{kPa}$;

Strength gradient, $k=1,2$ and $3 \mathrm{kPa} / \mathrm{m}$;

Soil unit weight, stiffener width and skirt thickness were kept constant as $\gamma^{\prime}=6$ 
$\mathrm{kN} / \mathrm{m}^{3}, b=0.17 \mathrm{~m}, h=0.25 \mathrm{~m}$ and $t=0.05 \mathrm{~m}$.

Combinations were chosen in order to cover a wide range of dimensionless parameter groups. Since no significant variation in soil flow mechanisms is found between $\alpha=0$ and 0.4 , only the results for smooth interface coefficient $(\alpha=0)$ are discussed in detail here. The critical depths are presented in Figure 10, with $H_{\mathrm{r}} / D$ (or $H_{\mathrm{c}} / D$ ) plotted against $s_{\mathrm{uHr} \text { (or Hc) }} / \gamma^{\prime} D$, where $s_{\mathrm{uHr} \text { (or Hc) }}$ is the intact shear strength at the corresponding depth of $H_{\mathrm{r}}$ or $H_{\mathrm{c}}$. The results varying with $b / D$ are fitted as below:

$$
\begin{aligned}
& \frac{H_{r}}{D}=\left(31.89 \frac{b}{D}+0.21\right)\left(\frac{s_{\mathrm{uH}_{\mathrm{r}}}}{\gamma^{\prime} D}\right)^{6.73 \frac{b}{D}+0.577} \\
& \frac{H_{\mathrm{C}}}{D}=\left(13.88 \frac{b}{D}+1.02\right)\left(\frac{s_{\mathrm{uH}_{\mathrm{c}}}}{\gamma^{\prime} D}\right)^{0.835}
\end{aligned}
$$

However, the Equations 1 and 2 are not explicit since $s_{\mathrm{uHr}}$ and $s_{\mathrm{uHc}}$ are functions of $H_{\mathrm{r}}$ and $H_{\mathrm{c}}$. Iterations are needed to obtain $H_{\mathrm{r}}$ and $H_{\mathrm{c}}$ values. With mathematical rearrangements, explicit alternatives of Equations 1 and 2 can be established as:

$$
\begin{aligned}
& \frac{H_{r}}{D}=\frac{C_{1} S^{C_{2}}}{1-\frac{k}{\gamma^{\prime}} C_{1} C_{2} S^{\left(C_{2}-1\right)}} \\
& \left(C_{1}=31.89 b / D+0.21, C_{2}=6.73 b / D+0.577, S=s_{\mathrm{um}} / \gamma^{\prime} D\right) \\
& \frac{H_{C}}{D}=\frac{C_{3} S^{C_{4}}}{1-\frac{k}{\gamma^{\prime}} C_{3} C_{4} S^{\left(C_{4}-1\right)}} \\
& \left(C_{3}=13.88 b / D+1.02, C_{4}=0.835\right)
\end{aligned}
$$




\section{Soil Strength Contour and Infill Soil Movement}

The distortion of the soil layers by the advancing smooth caisson is illustrated in Figure 11, plotting strength contours for the soil after a penetration of $d / t=140$, for the case of $k D / s_{\mathrm{um}}=2.26, s_{\mathrm{um}} / \gamma^{\prime} D=0.14$ (In Group VII, Table 1, case A) and $k D / s_{\mathrm{um}}=7.22, s_{\mathrm{um}} / \gamma^{\prime} D=0.028$ (In Group VII, Table 1 , case B). This shows that the soil outside the caisson is more or less undisturbed. The strength at any depth adjacent to the caisson is similar to the local shear strength $\left(s_{\mathrm{uo}}\right)$. The soil movement is mostly found to be restricted towards inside of the caisson, which was also observed by Andersen et al. (2005). The strength inside the caisson is degraded as the soil is sheared (e.g. see Figure 6). The softer soils trapped at the bottom stiffener base are from the upper layers (starting from the mudline, see Figure 11). However, due to the thin caisson wall $(D / t=226)$, negligible soil trapping can be seen beneath the advancing skirt tip.

The existing soil flow mechanism (Mechanism B in Figure 2; Erbrich and Hefer 2002) suggested that the friction between the infill soil and the inner skirt wall should be fully mobilized. This is not found here. Figure 12 shows the incremental soil flow velocity $v$ normalized by the skirt (or caisson) interval speed $v_{\text {skirt }}$ of the cases A and B in Figure 11. The ratio of $v / v_{\text {skirt }}=1$ indicates that the soil moves with a speed equivalent to that of the caisson. Clearly, the infill soils in the gaps between stiffeners move downward with the advancing caisson and mostly with a velocity similar of the skirt (i.e. $v / v_{\text {skirt }}=0.75 \sim 1$ ). Thus, there is no shearing between the infill soil and the inner skirt wall. The soil velocity field is more close to Mechanism A in Figure 2 (Erbrich and Hefer 2002), where the soil-soil sliding occurs on the vertical plane at the stiffener face. It should also be noted that the undrained shear strength mobilized along the soil-soil sliding plane is much lower than the intact strength of the soil, 
which is depicted through strength contours in Figure 11. No end bearing is mobilized at the base of any individual stiffeners above the bottom one.

\section{New Soil Flow Mechanisms and Penetration Resistance of Caisson with Stiffeners}

The newly proposed soil flow mechanisms are depicted in Figure 13 for caisson installation in clay with inside horizontal stiffeners. Based on this, the installation resistance can be expressed as the sum of three components:

$F=F_{\mathrm{o}}+F_{\mathrm{i}}+F_{\mathrm{tb}}$

As illustrated in Figure 13, the three components are: (1) resistance from the outside wall friction $\left(F_{\mathrm{o}}\right)$; (2) resistance from the inner skirt $\left(F_{\mathrm{i}}\right)$ and (3) end bearing resistance $\left(F_{\mathrm{tb}}\right)$. Due to the existence of stiffener, Component 2 involves the inner wall friction below the bottom stiffener $\left(F_{\mathrm{ib}}\right)$ and the frictional resistance above the bottom stiffener $\left(F_{\text {iabb }}\right)$ (i.e. $\left.F_{\mathrm{i}}=F_{\mathrm{ib}}+F_{\text {iabb }}\right)$. Component 3 includes end bearing at the tip of the skirt $\left(F_{\mathrm{t}}\right)$ and base of the bottom stiffener i.e. $F_{\mathrm{tb}}=F_{\mathrm{t}}+F_{\mathrm{b}}$. These components are discussed individually below.

\section{Component 1: Resistance from Outside Wall Friction $\left(F_{0}\right)$}

The outside wall friction occurs between the skirt (i.e. steel) and the adjacent soil with minimal soil disturbance (see Figure 11). The normalized penetration resistance $\left(F_{\mathrm{o}} / \alpha s_{\mathrm{uo}} A_{0}\right.$; where $\alpha$ is the interface roughness coefficient and $s_{\mathrm{uo}}$ is the average intact shear strength over the skirt penetration depth $d$ and $A_{0}$ is the outer wall area of the embedded skirt) plotted in Figure 14 for various $D / t$ and $k D / s_{\mathrm{um}}, s_{\mathrm{um}} / \gamma^{\prime} D$ displays a constant value of 1.0 during large penetration of the caissons skirt. This reflects that coefficient friction, $\alpha$, is the only influencing factor for $F_{0}$. 
This higher resistance forming a peak at shallow penetration depths indicates the effect of soil heaving outside the caisson, which augments the penetration resistance, since the area of the embedded skirt $\left(A_{0}\right)$ used in normalisation does not take the soil heave into account. Since in the field applications the penetration depths of $d /(b+t)$ are in the range of 20-266 (Andersen et al. 2005), the normalized peak resistance can be ignored as the resistance is still low at shallow penetration depth. As such, the resistance from the outside wall (component 1 in Equation 3) can be calculated as:

$F_{\mathrm{o}}=\alpha s_{\mathrm{uo}} A_{\mathrm{o}}=\alpha s_{\mathrm{uo}} \bullet \pi D d$

\section{Component 2: Resistance from Inner Skirt $\left(F_{\mathrm{i}}=F_{\mathrm{ib}}+F_{\text {iabb }}\right)$}

(a) Inner skirt wall friction below bottom stiffener $\left(F_{\mathbf{i b}}\right)$ : The frictional resistance along the inner skirt wall below the bottom stiffener is similar to the frictional resistance from the outer wall in Equation 4. This is evidenced in Figure 11 by the nearly intact soil strength contours along the inner skirt wall. The corresponding normalized resistances $\left(F_{\mathrm{ib}} / \alpha s_{\mathrm{uib}} A_{\mathrm{ib}}\right.$; where $S_{\mathrm{uib}}$ is the average intact undrained shear strength over the penetration depth below the base of the bottom stiffener, $A_{\mathrm{ib}}$ is the inner wall area of the embedded skirt below the bottom stiffener) also reach unity at deep penetrations. The plots of $F_{\mathrm{ib}} / \alpha s_{\mathrm{uib}} A_{\mathrm{ib}}$ vs $d /(t+b)$ are similar to the profiles in Figure 14, hence are not presented here. Thus, the inner friction below the bottom stiffener can be calculated as:

$F_{\text {ib }}=\alpha s_{\text {uib }} A_{\text {ib }}=\left\{\begin{array}{lll}\alpha s_{\text {uib }} \bullet \pi(D-2 t) d & \text { for } & d<w \\ \alpha s_{\text {uib }} \bullet \pi(D-2 t) w & \text { for } & d>w\end{array}\right.$

(b) Inner frictional resistance above bottom stiffener $\left(\boldsymbol{F}_{\text {iabb }}\right)$ : The inner frictional resistance above the bottom stiffener includes the friction between the embedded stiffener vertical faces and the adjacent soil (stiffener-soil interaction) and the friction 
between the trapped soils between embedded stiffener gaps and the adjacent soil (soilsoil interaction). Apparently, the resistance from the soil-soil interaction depends on the penetration depth relative to the critical depths $H_{\mathrm{r}}$ and $H_{\mathrm{c}}$. Rather than calculating the stiffener-soil interaction and soil-soil interaction separately, the lump-sum of inner frictional resistance is normalized as $F_{\text {iabb }} / s_{\text {uiab }} A_{\text {iab }}$, where $s_{\text {uiab }}$ is the average intact shear strength between bottom stiffener and limiting cavity depth $\left(H_{\mathrm{c}}\right)$ and $A_{\mathrm{iab}}$ is the corresponding inner wall area.

The effects of roughness $\alpha$, diameter ratio $D / t$ and relative strength $s_{\mathrm{um}} / \gamma^{\prime} D, s_{\mathrm{um}} / k D$ on the normalized resistance $F_{\text {iabb }} / s_{\text {uiab }} A_{\text {iab }}$ are plotted in Figure 15. It can be seen that the effect of $\alpha$ is minimal (see Figure 15a), while effects of $D / t$ and $s_{\mathrm{um}} / \gamma^{\prime} D, s_{\mathrm{um}} / k D$ are significant as these factors affect the critical depths $H_{\mathrm{r}}$ and $H_{\mathrm{c}}$ (Equations 1 and 2). For $d /(b+t) \geq 30$, the coefficient of inner friction $\left(\chi=F_{\text {iabb }} / s_{\text {uiab }} A_{\text {iab }}\right)$ reaches a limiting value. The limiting values of $\chi$ from all analyses display as a unique function of $\left(s_{\mathrm{um}} / \gamma^{\prime} D\right)\left(1+s_{\mathrm{um}} / k D\right)$ in Figure 15d. A best fit of the data allows $\chi$ to be approximated (with $\mathrm{R}^{2}=0.98$ ) as:

$\chi=0.472\left[\left(\frac{s_{\mathrm{um}}}{\gamma^{\prime} D}\right)\left(\frac{s_{\mathrm{um}}}{k D}+1\right)\right]^{0.528}+0.05 \leq 1$

Thus the inner friction above the bottom stiffener can be calculated as:

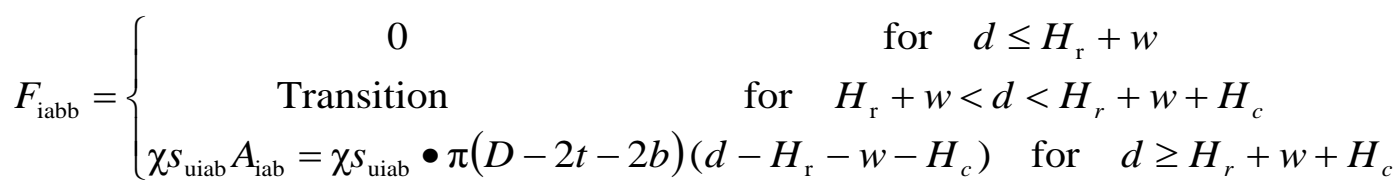

A linear interpolation should be sufficient in the transition phase. 


\section{Component 3: End Bearing Resistance $\left(F_{t b}=F_{t}+F_{b}\right)$}

(a) End bearing at the tip of the skirt $\left(F_{\mathrm{t}}\right)$ : The profiles of bearing factor, $N_{\mathrm{ct}}$, for a practical range of $\alpha$ and $k D / s_{\mathrm{um}}, s_{\mathrm{um}} / \gamma^{\prime} D$ are plotted in Figure 16 as a function of normalized skirt tip penetration depth $d / D$ instead of $d /(b+t)$ to be consistent with conventional analysis for bearing capacity factors of other foundations, with $N_{\text {ct }}$ calculated according to

$$
\begin{aligned}
& F_{\mathrm{t}}=\left(N_{\mathrm{ct}} s_{\mathrm{ut}}+\gamma^{\prime} d\right) A_{\mathrm{t}}=\left(N_{\mathrm{ct}} s_{\mathrm{ut}}+\gamma^{\prime} d\right) \bullet \frac{\pi\left(D^{2}-(D-2 t)^{2}\right)}{4} \\
& \approx\left(N_{\mathrm{ct}} s_{\mathrm{ut}}+\gamma^{\prime} d\right) \bullet \pi \mathrm{Dt}
\end{aligned}
$$

where $F_{\mathrm{t}}$ is the bearing resistance at the skirt tip, $s_{\mathrm{ut}}$ is the intact local undrained shear strength at the tip level and $A_{\mathrm{t}}$ is the area of the skirt tip. The approximation for the tip area in Equation 8 is due to high diameter ratio $D / t$. The influence of skirt tip roughness is considerable, particularly on deep bearing factors. For the range of roughness studied ( $\alpha=0 \sim 0.3$ ), the influence is less than $10 \%$ (see Figure 16a). The soil strength profiles, $k D / s_{\mathrm{um}}$ and $s_{\mathrm{um}} / \gamma^{\prime} D$, and the location of the bottom stiffener, $w$, have minimal effect on $N_{\mathrm{ct}}$ (see Figure 16b).

The results for skirt tip resistance are also compared with existing data for cone and caissons, as shown in Figure 16c. The comparison with cone response is due to the form of cavity expansion type failure mobilized beneath the skirt tip (see e.g. Figure 6), which is similar to the mechanism associated with a cone penetration ( $\mathrm{Lu}$ et al. 2004). Figure 16c shows the reported bearing factor, $N_{\mathrm{kt}}$, profiles for smooth and rough cones from LDFE analyses (Lu et al. 2004; Liyanapathirana 2009), a smooth skirted foundation ( $D=12 \mathrm{~m}$ ) from small strain analyses (Mana et al. 2012), and for an unstiffened caisson with $\alpha=0.3$ from LDFE analyses (Zhou and Randolph 2006). The difference between cone and caisson penetration responses is mainly due to the 
geometries of cavity expansion in soil: axisymmetric for cone and near plane strain for caisson skirt. Nevertheless, for a smooth interface ( $\alpha=0)$, the results for a cone (Lu et al. 2004; Liyanapathirana 2009) and skirted foundation (Mana et al. 2012) agree very well. The results from this study (for $\alpha=0.1 \sim 0.3$ ) are nicely bounded by the reported cone factors for $\alpha=0$ and 1 , and agree well with the caisson factors for $\alpha$ $=0.3\left(s_{\mathrm{u}}=4+1.5 \mathrm{z} \mathrm{kPa}, \gamma^{\prime}=6.5 \mathrm{kN} / \mathrm{m}^{3}, D=2 \mathrm{~m}\right.$; Zhou and Randolph 2006). Based on the current results, fitted design curves for caisson skirt tip end bearing factor can be expressed as

$$
N_{\mathrm{ct}}=\left\{6.36+4.768\left(\frac{d}{D}\right)^{0.2}\right\}(1+\alpha)^{0.2} \leq 13.5 \text { for } \alpha=0 \sim 0.3
$$

(b) End bearing at the base of the bottom stiffener $\left(\boldsymbol{F}_{\mathbf{b}}\right)$ : The profiles of bearing capacity factor of the bottom stiffener $N_{\mathrm{cb}}$ are plotted in Figure 17, with $N_{\mathrm{cb}}$ calculated according to

$$
\begin{aligned}
& F_{\mathrm{b}}=\left(N_{\mathrm{cb}} S_{\mathrm{ub}}+\gamma^{\prime} d_{\mathrm{b}}\right) A_{\mathrm{b}}=\left(N_{\mathrm{cb}} S_{\mathrm{ub}}+\gamma^{\prime} d_{\mathrm{b}}\right) \cdot \frac{\pi\left((D-2 t)^{2}-(D-2 t-2 b)^{2}\right)}{4} \\
& \approx\left(N_{\mathrm{cb}} S_{\mathrm{ub}}+\gamma^{\prime} d_{\mathrm{b}}\right) \cdot \pi \mathrm{D} b
\end{aligned}
$$

where $F_{\mathrm{b}}$ is the bearing resistance at the base of the bottom stiffener base, $s_{\mathrm{ub}}$ is the intact local undrained shear strength at the base level and $A_{\mathrm{b}}$ is the base area of the ring stiffener, again the approximation of $A_{\mathrm{b}}$ is due to the high diameter ratios of $D / t$ and $D /(t+b)$. The effects of roughness $\alpha$, bottom stiffener location $w / b$ and relative soil strength $s_{\mathrm{um}} / \gamma^{\prime} D, s_{\mathrm{um}} / k D$ all seem insignificant. Similar to $N_{\mathrm{ct}}$, design chart for $N_{\mathrm{cb}}$ is shown in Figure 17c. The bottom stiffener can be seen as similar to half of a fullflow penetrometer, T-bar, as the soil flow is directed towards the soil surface and flows around the stiffener with further penetration of the caisson. Martin and 
Randolph (2006) gave detailed limit analyses for a cylindrical object undergoing lateral translation in undrained uniform clay. Aubeny et al. (2005) presented bearing capacity factors for a deep-penetrating (up to 5 diameters) horizontal cylinder in nonhomogeneous clays by FE analysis and plasticity theory. The trench in the wake of the advancing cylinder was assumed as fully open. However, in all cases, the soil weight and hence surface heaving was ignored. White et al. (2010) reported T-bar factors at shallow embedment in uniform clay from LDFE analyses.

Figure 17c shows the comparison of bearing factors of caisson bottom stiffener $N_{\mathrm{cb}}$ and T-bar (or cylindrical bar) $N_{\text {T-bar. }}$ The penetration depth of the bottom stiffener, $d_{\mathrm{b}}$, is normalized by the caisson diameter, $D$. Aubeny et al.'s $N_{\text {T-bar }}$ factors were increased by $10 \%$ uniformly over the full penetration depths, accounting for the effect of soil heaving, as suggested by Randolph and White (2008). The profiles of $N_{\mathrm{cb}}$ (for $\alpha=$ $0.1 \sim 0.3$ ) are bracketed by the curves of T-bar factors for $\alpha=0$ and 1 . The factors reported by White et al. (2010) lie above the curve for $\alpha=1$, which may be due to the considered uniform clay, hence the absence of the effect of soil strength nonhomogeneity, where softer soil can be trapped underneath the penetrating object. The results of $N_{\mathrm{cb}}$ from this study can be approximated (neglecting very small effect of $\alpha$ ) as

$$
N_{\text {cb }}=5.14+2.5\left(\frac{d}{D}\right)^{0.53} \leq 10.5 \text { for } \alpha=0 \sim 0.3
$$

\section{Total Resistance of Caisson during Installation}

For stiffened caisson penetration in clay, the total resistance during installation can be calculated following Equation 3 as:

$$
F=\alpha s_{\mathrm{uo}} A_{\mathrm{o}}+\alpha s_{\mathrm{uib}} A_{\mathrm{ib}}+\chi s_{\mathrm{uiab}} A_{\mathrm{iab}}+\left(N_{\mathrm{ct}} s_{\mathrm{ut}}+\gamma^{\prime} d_{\mathrm{t}}\right) A_{\mathrm{t}}+\left(N_{\mathrm{cb}} s_{\mathrm{ub}}+\gamma^{\prime} d_{\mathrm{b}}\right) A_{\mathrm{b}}
$$


For unstiffened caisson penetration, components $2 \mathrm{a}: F_{\mathrm{ib}}$ and $2 \mathrm{~b}$ : $F_{\text {iabb }}$ will merge together representing resistance from the inside wall friction $F_{\mathrm{i}}$; and component $3 \mathrm{~b}: F_{\mathrm{b}}$ will be zero. Thus, Equation 12 can be simplified as:

$$
F=F_{\mathrm{o}}+F_{\mathrm{i}}+F_{\mathrm{tb}}=\alpha \mathrm{s}_{\mathrm{uo}} A_{\mathrm{o}}+\alpha \mathrm{s}_{\mathrm{uo}} A_{\mathrm{i}}+\left(N_{\mathrm{ct}} s_{\mathrm{ut}}+\gamma^{\prime} d_{\mathrm{t}}\right) A_{\mathrm{t}}
$$

\section{Summary of Design Procedure and Validation}

\section{Summary of Design Procedure}

For stiffened caissons in NC clay and lightly overconsolidated clay, based on the discussion on individual component in the previous section, a design procedure is proposed to predict installation resistance, hence the underpressure required to achieve the designed penetration depth. The procedure is outlined below.

1. Determine representative values of the soil parameters $s_{\mathrm{um}}, k$ and $\gamma^{\prime}$.

2. Take all geometric parameters of the stiffened caisson, $D, t, b, s, h$, as primary input.

3. Evaluate the dimensionless ratios $s_{\mathrm{um}} / \gamma^{\prime} D, k D / s_{\mathrm{um}}, D / t, \mathrm{~s} / \mathrm{h}$ and $b / t$.

4. Calculate the critical depths of rotational flow, $H_{\mathrm{r}}$, and cavity depth, $H_{\mathrm{c}}$, using Equations 1 and 2, respectively.

5. Use Equation 3 or 12 to evaluate the penetration resistance, calculating

- Component $1, F_{0}$, from Equation 4

- $\quad$ Component 2, $F_{\mathrm{i}}$, from Equations 5 7

- $\quad$ Component 3, $F_{\text {tb }}$, from Equations 8 11 


\section{Validation}

Figures 18a and 18b show comparisons among the measured field data (Erbrich and Hefer 2002) and centrifuge test data (Gaudin et al. 2014), numerical result from this study and predictions using the proposed design approach. It can be seen that the estimated profiles agree well with the measured data for the full penetration depths, including self-weight and suction installation.

The sensitivity of each component contributing to the total resistance is depicted in Figure 18c, reflecting the domination of end bearing of the bottom stiffener base $F_{\mathrm{b}}$ (component 3) and outer wall friction $F_{\mathrm{o}}$ (component 1).

Comparison is also made for an unstiffened caisson. Equation 13 is used for assessing penetration resistance of a caisson of $D=4 \mathrm{~m}, t=0.04 \mathrm{~m}$ installing in clay with $s_{\mathrm{u}}=4+1.5 \mathrm{z} \mathrm{kPa}(\alpha=0.3)$. Figure 18d compares the predicted components using the proposed approach and the LDFE results reported by Zhou and Randolph (2006). Very good agreement is achieved.

\section{Concluding Remarks}

This paper has reported the results from LDFE analysis using the RITSS method in AFENA, simulating continuous penetration of stiffened caissons from the seabed surface. The extensive investigation on normally consolidated and lightly overconsolidated clays encompassed the relevant range of normalized soil properties; skirt- and stiffener-soil interface roughness coefficient; width, spacing and height of stiffeners; distance of the bottom stiffener from the skirt tip; diameter of the caisson and thickness of the skirt wall. 
A new mechanism-based design approach was proposed for estimating installation resistance corresponding to the targeted final penetration depth. The key features of the proposed mechanism are: (i) when the bottom stiffener penetration reaches $H_{\mathrm{r}}$, rotational flow around the bottom stiffener and soil scraping from the second bottom stiffener starts to occur, leading to fill in the gap between these two stiffeners; (ii) when the second bottom stiffener penetration reaches $H_{\mathrm{c}}$, soil starts to flow into the gap above that stiffener. The critical depths $H_{\mathrm{r}}$ and $H_{\mathrm{c}}$ can be calculated explicitly using Equations 1a and 2a.

The simple expression of stiffened skirt penetration resistance (Equation 12) includes three components: (i) resistance from outside wall friction; (ii) resistance from inside skirt friction; and (iii) end bearing from skirt tip and bottom stiffener. Equation 12 has been validated against field test data and centrifuge test data, with excellent agreement obtained.

\section{Acknowledgements}

The research presented here was undertaken with support from National Nature Science Foundation of China (No. U1134207 and B13024), the Department of Industry, Innovation, Science, Research and Tertiary Education (DIISRTE) Australia China Science and Research Fund (Group Mission ACSRF00300) and the Australian Research Council (ARC) Discovery Grant (DP1096764). The first author is an ARC Discovery Early Career Researcher Award (DECRA) Fellow and is supported by the ARC Project DE140100903. The work forms part of the activities of the Centre for

Offshore Foundation Systems (COFS), currently supported as a node of the Australian Research Council Centre of Excellence for Geotechnical Science and Engineering and 
as a Centre of Excellence by the Lloyd's Register Foundation. This support is gratefully acknowledged, as is the benefit of discussion with Dr Long Yu, Dr Dong Wang and Dr Xu Li. 


\section{References}

Andersen, K., Murff, J., Randolph, M., Clukey, E., Erbrich, C., Jostad, H., Hansen, B., Aubeny, C., Sharma, P., and Supachawarote, C. (2005). "Suction anchors for deepwater applications.” Proc., 1st Int. Symp. on Frontiers in Offshore Geotechnics (ISFOG), Perth, 3-30.

API (2007). API Recommended practice 2A-WSD. American Petroleum Institute.

Aubeny, C., Shi, H., and Murff, J. (2005). “Collapse loads for a cylinder embedded in trench in cohesive soil.” International Journal of Geomechanics, 5(4), 320325.

Carter, J., and Balaam, N. (1995). “AFENA users’ manual.” Centre for Geotechnical Research, Department of Civil Engineering, University of Sydney, Australia.

Chen, W., and Randolph, M. (2007). "External radial stress changes and axial capacity for suction caissons in soft clay.” Geotechnique, 57(6), 499-511.

Chen, W., Zhou, H., and Randolph, M. (2009). "Effect of installation method on external shaft friction of caissons in soft clay.” Journal of Geotechnical and Geoenvironmental Engineering, 135(5), 605-615.

Dendani, H. (2003). "Suction anchors: some critical aspects for their design and installation in clayey soils.” Proc., Offshore Technology Conference, Offshore Technology Conference, Houston, OTC 15376.

Dendani, H., and Colliat, J.-L. (2002). “Girassol: design analysis and installation of the suction anchors." Proc., Offshore Technology Conference, Offshore Technology Conference, Houston, OTC 14209. 
Erbrich, C., and Hefer, P. (2002). "Installation of the Laminaria suction piles - a case history.” Proc., Offshore Technology Conference, Offshore Technology Conference, Houston, OTC 14240.

Gaudin, C., O’, Loughlin, C. D., Hossain, M. S., Randolph, M. F., and Colliat, J.-L. (2014). "Installation of suction caissons in Gulf of Guinea clay.” Proc., 8th Int. Conf. on Physical Modelling in Geotechnics, Perth, 1, 493-499.

Ghosh, S., and Kikuchi, N. (1991). “An arbitrary Lagrangian-Eulerian finite element method for large deformation analysis of elastic-viscoplastic solids.” Computer Methods in Applied Mechanics and Engineering, 86(2), 127-188.

Herrmann, L. R. (1978). “Finite element analysis of contact problems.” Journal of the Engineering Mechanics Division, 104(5), 1043-1057.

Hossain, M., Lehane, B., Hu, Y., and Gao, Y. (2012). "Soil flow mechanisms around and between stiffeners of caissons during installation in clay.” Canadian Geotechnical Journal, 49(4), 442-459.

House, A., and Randolph, M. (2001). “Installation and pull-out capacity of stiffened suction caissons in cohesive sediments.” Proc., 11th International Offshore and Polar Engineering Conference, 2, 574-580.

Hu, Y., and Randolph, M. (1998). “A practical numerical approach for large deformation problems in soil.” International Journal for Numerical and Analytical Methods in Geomechanics, 22(5), 327-350.

Liyanapathirana, D. (2009). “Arbitrary Lagrangian Eulerian based finite element analysis of cone penetration in soft clay.” Computers and Geotechnics, 36(5), 851-860. 
Lu, Q., Randolph, M., Hu, Y., and Bugarski, I. (2004). “A numerical study of cone penetration in clay.” Geotechnique, 54(4), 257-267.

Mana, D. S. K., Gourvenec, S. M., and Randolph, M. F. (2013). "Experimental investigation of reverse end bearing of offshore shallow foundations.” Canadian Geotechnical Journal, 139(9), 1022-1033.

Martin, C., and Randolph, M. (2006). "Upper-bound analysis of lateral pile capacity in cohesive soil.” Geotechnique, 56(2), 141-146.

Randolph, M. F., and House, A. R. (2002). “Analysis of suction caisson capacity in clay.” Proc., Offshore Technology Conference, Offshore Technology Conference, Houston, OTC 14236.

Randolph, M., O’Neill, M., Stewart, D., and Erbrich, C. (1998). "Performance of suction anchors in fine-grained calcareous soils.” Proc., Offshore Technology Conference, Offshore Technology Conference, Houston, OTC 8831.

Randolph, M., and White, D. (2008). “Upper-bound yield envelopes for pipelines at shallow embedment in clay.” Geotechnique, 58(4), 297-301.

Sloan, S., and Booker, J. (1986). "Removal of singularities in Tresca and MohrCoulomb yield functions.” Communications in Applied Numerical Methods, 2(2), 173-179.

Vásquez, L. F. G., Maniar, D. R., and Tassoulas, J. L. (2010). “Installation and axial pullout of suction caissons: numerical modelling.” Journal of Geotechnical and Geoenvironmental Engineering, ASCE, 136(8), 1137-1147.

Westgate, Z., Tapper, L., Lehane, B., and Gaudin, C. (2009). "Modelling the installation of stiffened caissons in overconsolidated clay.” Proc., ASME 2009 
28th International Conference on Ocean, Offshore and Arctic Engineering, American Society of Mechanical Engineers, 119-126.

White, D., Gaudin, C., Boylan, N., and Zhou, H. (2010). “Interpretation of T-bar penetrometer tests at shallow embedment and in very soft soils.” Canadian Geotechnical Journal, 47(2), 218-229.

Zhou, H., and Randolph, M. F. (2006). "Large deformation analysis of suction caisson installation in clay.” Canadian Geotechnical Journal, 43(12), 1344-1357.

Zhou, H., and Randolph, M. F. (2009). "Resistance of full-flow penetrometers in ratedependent and strain-softening clay.” Geotechnique, 59(2), 79-86. 
Table 1. Summary of LDFE analyses performed on non-homogeneous clay: soil

failure mechanisms

\begin{tabular}{|c|c|c|c|c|c|c|c|c|}
\hline Analysis & $D / t$ & $b / t$ & $s / h$ & $w / b$ & $\alpha$ & $k D / s_{\mathrm{um}}$ & $s_{\mathrm{um}} / \gamma^{\prime} D$ & Notes \\
\hline Group I & - & $\begin{array}{l}2.0 \\
4.0\end{array}$ & 2.0 & $\begin{array}{l}5.3 \\
2.7\end{array}$ & 0.3 & - & - & $\begin{array}{l}\text { Comparison with centrifuge } \\
\text { data of Hossain et al. (2012) }\end{array}$ \\
\hline Group II & 226 & 3.4 & 4.0 & 5.9 & 0.2 & 2.26 & 0.070 & $\begin{array}{l}\text { General soil flow } \\
\text { mechanisms }\end{array}$ \\
\hline Group III & $\begin{array}{c}90 \\
240\end{array}$ & 3.4 & 4.0 & 5.9 & 0.2 & $\begin{array}{l}0.45 \\
1.20\end{array}$ & $\begin{array}{l}0.370 \\
0.140\end{array}$ & $\begin{array}{l}\text { Investigation of the effect of } \\
\text { caisson diameter }\end{array}$ \\
\hline Group IV & 160 & $\begin{array}{l}3.4, \\
7.0\end{array}$ & 4.0 & $\begin{array}{l}2.0 \\
7.0\end{array}$ & $\begin{array}{l}0.0 \\
0.2, \\
0.4\end{array}$ & 0.80 & 0.210 & $\begin{array}{l}\text { Investigation of the effect of } \\
\text { stiffener width and } \\
\text { coefficient of friction }\end{array}$ \\
\hline Group V & 226 & 3.4 & 4.0 & 5.2 & 0.2 & 22.60 & 0.007 & $\begin{array}{l}\text { Investigation of the effect of } \\
\text { normalized soil strength and } \\
\text { its non-homogeneity }\end{array}$ \\
\hline Group VI & $\begin{array}{l}90,120 \\
160,226 \\
\quad 300\end{array}$ & 3.4 & 4.0 & 5.2 & 0.2 & $*$ & $*$ & $\begin{array}{l}\text { Investigation for depth of } \\
\text { rotational flow and limiting } \\
\text { cavity depth }\end{array}$ \\
\hline \multicolumn{9}{|c|}{$\begin{array}{l}* \text { For each } D / t \text {, a total of } 9 \text { strength profiles were explored varying } s_{\text {um }} \text { and } k \text { as } s_{\text {um }}=0.5,5 \text { and } 10 \mathrm{kPa} \\
\text { and } k=1,2 \text { and } 3 \mathrm{kPa} / \mathrm{m}\end{array}$} \\
\hline
\end{tabular}


Table 2. Summary of LDFE analyses performed on non-homogeneous clay:

penetration resistance

\begin{tabular}{|c|c|c|c|c|c|c|c|c|}
\hline Analysis & $D / t$ & $b / t$ & $s / h$ & $w / b$ & $\alpha$ & $k D / s_{\mathrm{um}}$ & $s_{\mathrm{um}} / \gamma^{\prime} D$ & Notes \\
\hline Group I-A & 100 & 6.3 & 19.0 & 8.0 & 0.3 & 7.22 & 0.030 & $\begin{array}{l}\text { Comparison with centrifuge } \\
\text { data of Gaudin et al. (2014) }\end{array}$ \\
\hline Group I-B & 226 & 3.4 & 3.0 & 5.2 & 0.2 & 3.16 & 0.150 & $\begin{array}{l}\text { Comparison with centrifuge } \\
\text { data of Westgate et al. (2009) }\end{array}$ \\
\hline Group II-A & $\begin{array}{c}90,120 \\
150\end{array}$ & 3.4 & 12.0 & 11.8 & 0.2 & $\begin{array}{l}2.25 \\
3.00 \\
3.75\end{array}$ & $\begin{array}{l}0.074 \\
0.055 \\
0.044\end{array}$ & $\begin{array}{l}\text { Investigation of the effect of } \\
\text { caisson diameter on outer } \\
\text { friction and inner friction } \\
\text { below bottom stiffener }\end{array}$ \\
\hline Group II-B & 90 & 3.4 & 12.0 & 11.8 & 0.2 & $\begin{array}{l}2.25 \\
4.50 \\
6.75 \\
0.90 \\
0.45\end{array}$ & $\begin{array}{l}0.074, \\
0.074, \\
0.074, \\
0.185, \\
0.370\end{array}$ & $\begin{array}{l}\text { Investigation of the effect of } \\
\text { soil strength on outer friction } \\
\text { and inner friction below } \\
\text { bottom stiffener }\end{array}$ \\
\hline Group III-A & 90 & 3.4 & 12.0 & 11.8 & $\begin{array}{l}0.0 \\
0.2 \\
0.4\end{array}$ & 2.25 & 0.074 & $\begin{array}{l}\text { Investigation of the effect of } \\
\text { coefficient of friction on inner } \\
\text { friction above bottom } \\
\text { stiffener }\end{array}$ \\
\hline Group III-B & $\begin{array}{c}80,160 \\
240\end{array}$ & 3.4 & 12.0 & 11.8 & 0.2 & $\begin{array}{l}2.00 \\
4.00 \\
6.00\end{array}$ & $\begin{array}{l}0.083, \\
0.041 \\
0.028\end{array}$ & $\begin{array}{l}\text { Investigation of the effect of } \\
\text { caisson diameter on inner } \\
\text { friction above bottom } \\
\text { stiffener }\end{array}$ \\
\hline Group III-C & 90 & 3.4 & 4.0 & 5.9 & 0.2 & $\begin{array}{l}9.00 \\
0.90 \\
0.45\end{array}$ & $\begin{array}{l}0.019, \\
0.185, \\
0.370\end{array}$ & $\begin{array}{l}\text { Investigation of the effect of } \\
\text { soil strength on inner friction } \\
\text { above bottom stiffener }\end{array}$ \\
\hline Group III-D & $\begin{array}{l}80, \quad 120 \\
160,240 \\
300\end{array}$ & 3.4 & 12.0 & 11.8 & 0.2 & $* *$ & $* *$ & $\begin{array}{l}\text { Investigation of the effect of } \\
\text { coefficient of inner friction } \\
\text { above bottom stiffener }\end{array}$ \\
\hline Group IV & 90 & 3.4 & 12.0 & $\begin{array}{l}5.9, \\
11.8, \\
17.6, \\
\infty\end{array}$ & $\begin{array}{l}0.0 \\
0.1 \\
0.2 \\
0.3\end{array}$ & $\begin{array}{l}0.90 \\
1.80 \\
1.35 \\
0.45\end{array}$ & $\begin{array}{l}0.185, \\
0.370\end{array}$ & $\begin{array}{l}\text { Investigation of the effect of } \\
\text { soil strength, distance of } \\
\text { bottom stiffener from skirt tip } \\
\text { and coefficient of friction on } \\
\text { skirt tip bearing capacity } \\
\text { factor, } N_{\mathrm{ct}} \text {, and bottom } \\
\text { stiffener bearing capacity } \\
\text { factor, } N_{\mathrm{cb}}\end{array}$ \\
\hline
\end{tabular}


Number of Figure: 18

Figure 1. General view of suction caisson installation: (a) Schematic diagram of stiffened caisson installing in non-homogeneous clay; (b) Initial mesh for LDFE analysis (axes in meter)

Figure 2. Soil flow mechanisms for stiffened caisson installation proposed by Erbrich \& Hefer (2002) and Dendani (2003)

Figure 3. Significant overestimation related to uncertainty in soil failure mechanisms for stiffened caissons: (a) Measured vs predicted suction pressures (Laminaria: Erbrich and Hefer 2002); (b) Measured vs predicted suction pressures (Girassol: Dendani and Colliat 2002; Dendani 2003)

Figure 4. Comparison of soil flow mechanisms between LDFE analysis and centrifuge test data reported by Hossain et al. (2014) (plane strain, $b / t=2.0$ and 4.0, $s / h=2.0, w / b=5.3$ and 2.7, $\alpha=0.3$; Group I, Table 1): (a) $d / t=28$ (axes in $\mathrm{mm}$ ); (b) $d / t=41$ (axes in $\mathrm{mm}$ )

Figure 5. Comparison of installation resistance between LDFE analysis and centrifuge test data: (a) Comparison with data reported by Gaudin et al. (2014) $(D / t=100, b / t=6.3, s / h=19.0, w / b=8.0, \alpha=0.3$, $k D / s_{\mathrm{um}}=7.22, s_{\mathrm{um}} / \gamma^{\prime} D=0.030 ;$ Group I-A, Table 2); (b) Comparison with data reported by Westgate et al. (2009) $(D / t=$ 226, $b / t=3.4, s / h=3.0, w / b=5.2, \alpha=0.2, k D / s_{\mathrm{um}}=3.16, s_{\mathrm{um}} / \gamma^{\prime} D=$ 0.150; Group I-B, Table 2)

Figure 6. Soil flow mechanisms for stiffened caisson penetration in nonhomogeneous clay $(D / t=226, b / t=3.4, s / h=4.0, w / b=5.9, \alpha=0.2$, $k D / s_{\mathrm{um}}=2.26, s_{\mathrm{um}} / \gamma^{\prime} D=0.070$; Group II, Table 1): (a) $d / t=60$ (axes in $\mathrm{m}$ ); (b) $d / t=120$ (axes in $\mathrm{m}$ )

Figure 7. Effect of caisson diameter on soil flow mechanisms at $d / t=90(b / t=$ 3.4, $s / h=4.0, w / b=5.9, \alpha=0.2$; Group III, Table 1): (a) $D / t=90$ $\left(k D / s_{\mathrm{um}}=0.45, s_{\mathrm{um}} / \gamma^{\prime} D=0.370\right.$; axes in m); (b) $D / t=240\left(\mathrm{kD} / \mathrm{s}_{\mathrm{um}}=\right.$ 1.20, $s_{\mathrm{um}} / \gamma^{\prime} D=0.140$; axes in $\mathrm{m}$ )

Figure 8. Effect of stiffener width on soil flow mechanisms at $d / t=102(D / t=$ 160, $\mathrm{s} / \mathrm{h}=4.0, \alpha=0.2, \mathrm{kD} / \mathrm{s}_{\mathrm{um}}=0.80, \mathrm{~s}_{\mathrm{um}} / \gamma^{\prime} D=0.210$; Group IV, Table 1): (a) $b / t=3.4$ (axes in $\mathrm{m}$ ), (b) $b / t=7.0$ (axes in $\mathrm{m}$ )

Figure 9. Effect of soil strength non-homogeneity and normalized strength on soil flow mechanisms $\left(k D / s_{\mathrm{um}}=22.60, s_{\mathrm{um}} / \gamma^{\prime} D=0.007, D / t=226\right.$, $b / t=3.4, s / h=4.0, w / b=5.2, \alpha=0.2$; Group V, Table 1): (a) $d / t=$ 29 (axes in $\mathrm{m})$; (b) $d / t=100$ (axes in $\mathrm{m}$ )

Figure 10. Design charts for estimating critical depths of soil infill in gaps between embedded stiffeners (Group VI, Table 1): (a) Depth of rotational flow $\left(H_{\mathrm{r}}\right)$; (b) Limiting cavity depth $\left(H_{\mathrm{c}}\right)$ 
Figure 11. Strength contours for stiffened caisson penetration in nonhomogeneous clay: (a) Case A: $d / t=140, D / t=226, b / t=3.4, s / h=$ 4.0, $w / b=5.2, \alpha=0.2, s_{\mathrm{u}}=5+1 \mathrm{z} \mathrm{kPa}$ (in Group VI, Table 1); (b) Case B: $d / t=238, D / t=100, b / t=7.3, s / h=19.0, w / b=8.0, \alpha=0.3$, $s_{\mathrm{u}}=1.33+1.2 \mathrm{z} \mathrm{kPa}($ Group I-A, Table 1$)$

Figure 12. Soil vertical flow velocity contours for stiffened caisson penetration in clay

Figure 13. A new mechanism for stiffened caisson installation in clay

Figure 14. Friction along outer periphery of caisson $\left(F_{0}\right)$ : (a) Effect of normalized caisson diameter, $D / t(b / t=3.4, s / h=12.0, w / b=11.8, \alpha$ $=0.2, \mathrm{kD} / \mathrm{s}_{\mathrm{um}}=2.25 \sim 3.75, s_{\mathrm{um}} / \gamma^{\prime} D=0.074 \sim 0.044$; Group II-A, Table 2); (b) Effect of soil strength non-homogeneity, $\mathrm{kD} / \mathrm{s}_{\mathrm{um}}$, and normalized strength, $s_{\mathrm{um}} / \gamma^{\prime} D(D / t=90, b / t=3.4, s / h=12.0, w / b=$ 11.8, $\alpha=0.2$; Group II-B, Table 2)

Figure 15. Inner friction above bottom stiffener $\left(F_{\text {iabb }}\right)$ (a) Effect of coefficient of friction, $\alpha(D / t=90, b / t=3.4, s / h=12.0, w / b=11.8$, $k D / s_{\mathrm{um}}=2.25, s_{\mathrm{um}} / \gamma^{\prime} D=0.074$; Group III-A, Table 2); (b) Effect of normalized caisson diameter, $D / t(b / t=3.4, s / h=12.0, w / b=11.8, \alpha$ $=0.2, k D / s_{\mathrm{um}}=2.00 \sim 6.00, s_{\mathrm{um}} / \gamma^{\prime} D=0.083 \sim 0.028$; Group III-B, Table 2); (c) Effect of soil strength non-homogeneity, $\mathrm{kD} / \mathrm{s}_{\mathrm{um}}$, and normalized strength, $s_{\mathrm{um}} / \gamma^{\prime} D(D / t=90, b / t=3.4, s / h=4.0, w / b=5.9$, $\alpha=0.2$; Group III-C, Table 2); (d) Design chart for coefficient of inner friction above bottom stiffener, $\chi(D / t=80 \sim 300, b / t=3.4, s / h$ $=$ 12.0, $w / b=11.8, \alpha=0.2$; Group III-D, Table 2)

Figure 16. End bearing factor at skirt tip $\left(N_{\mathrm{ct}}\right)$ : (a) Effect of coefficient of friction, $\alpha\left(D / t=90, b / t=3.4, s / h=12.0, w / b=11.8, k D / s_{\mathrm{um}}=0.90\right.$, $s_{\mathrm{um}} / \gamma^{\prime} D=0.185$; in Group IV, Table 2); (b) Effect of distance from bottom stiffener to skirt tip, $w / b$, and soil strength nonhomogeneity, $k D / s_{\mathrm{um}}$ and normalized strength, $s_{\mathrm{um}} / \gamma^{\prime} D(D / t=90$, $b / t=3.4, s / h=12.0, \alpha=0.2$; in Group IV, Table 2); (c) Design chart for $N_{\text {ct }}$

Figure 17. End bearing factor at bottom stiffener base $\left(N_{\mathrm{cb}}\right)$ : (a) Effect of coefficient of friction, $\alpha(D / t=90, b / t=3.4, s / h=12.0, w / b=11.8$, $k D / s_{\mathrm{um}}=0.90, s_{\mathrm{um}} / \gamma^{\prime} D=0.185$; in Group IV, Table 2); (b) Effect of distance from bottom stiffener to skirt tip, $w / b$, and soil strength non-homogeneity, $k D / s_{\mathrm{um}}$ and normalized strength, $s_{\mathrm{um}} / \gamma^{\prime} D(D / t=$ 90, $b / t=3.4, s / h=12.0, \alpha=0.2$; in Group IV, Table 2); (c) Proposed design chart for $N_{\mathrm{cb}}$

Figure 18. Comparison between prediction using proposed mechanism-based design approach and measured and computed data: (a) Comparison with field installation data reported by Erbrich and Hefer (2002); (b) Comparison with centrifuge test data reported by Gaudin et al. (2014); (c) Sensitivity of different components 
(stiffened caisson) - predicted results; (d) Sensitivity of different components (unstiffened caisson) - predicted results 


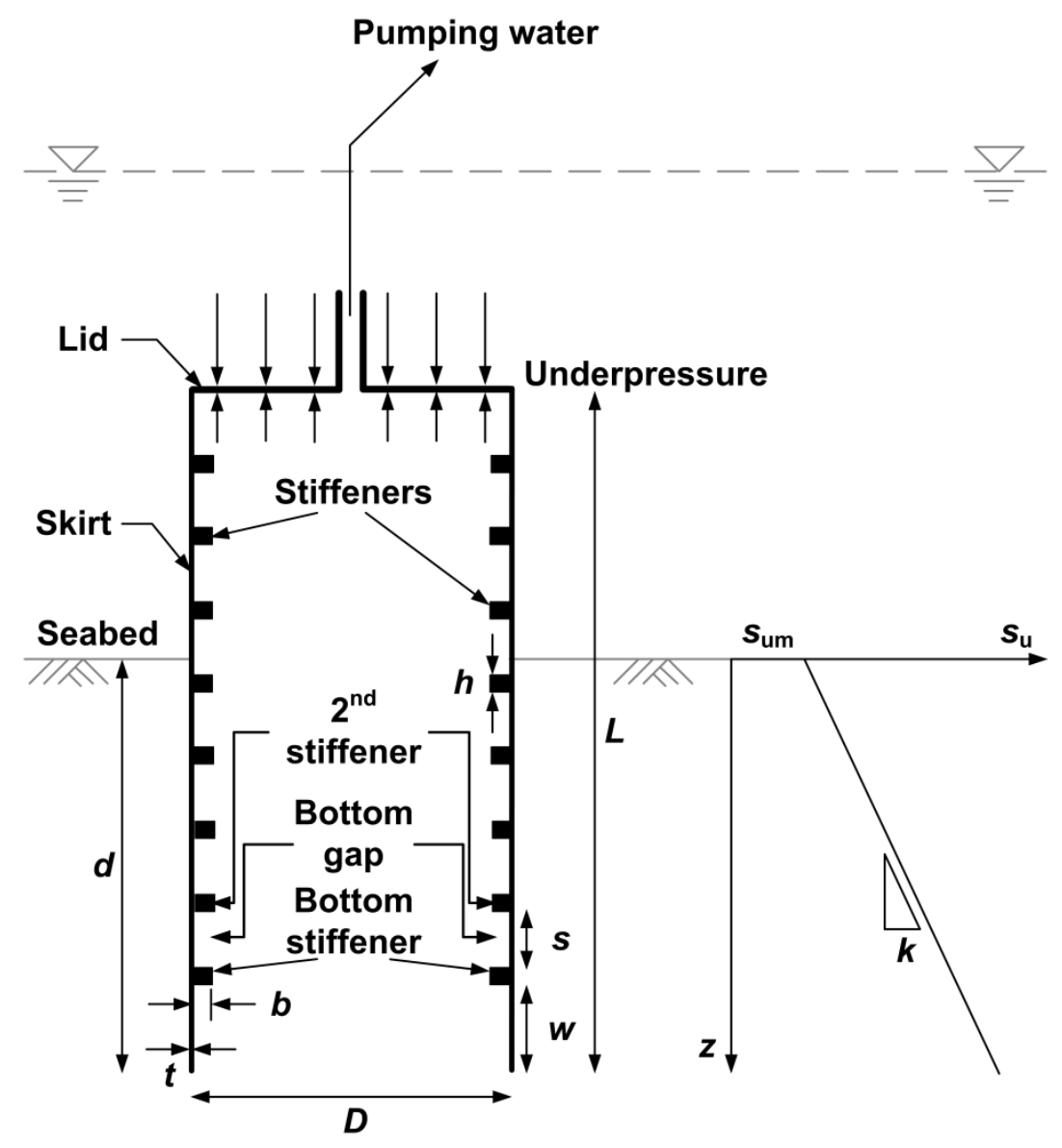

1(a) Schematic diagram of stiffened caisson installing in non-homogeneous clay 

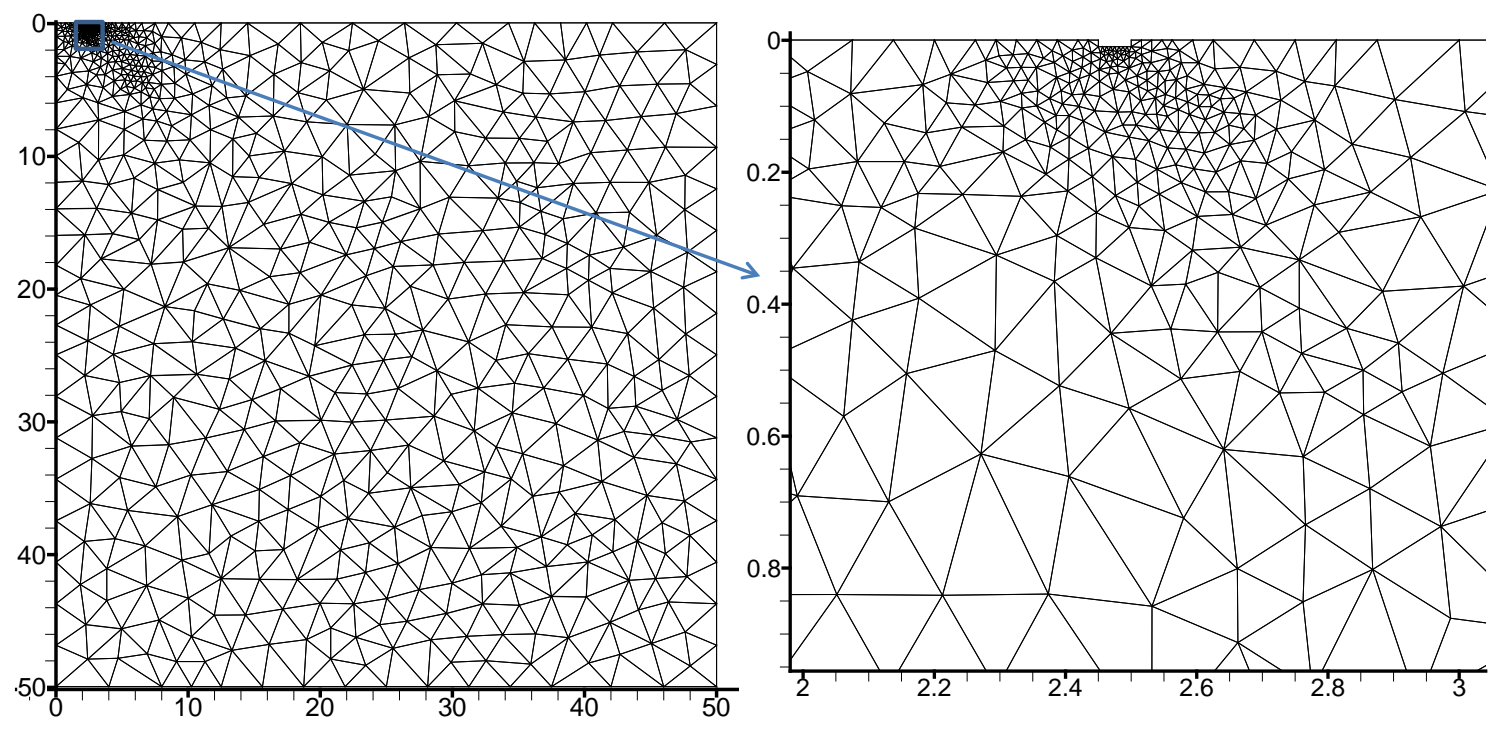

1(b) Initial mesh for LDFE analysis (axes in meter)

Figure 1. General view of suction caisson installation 


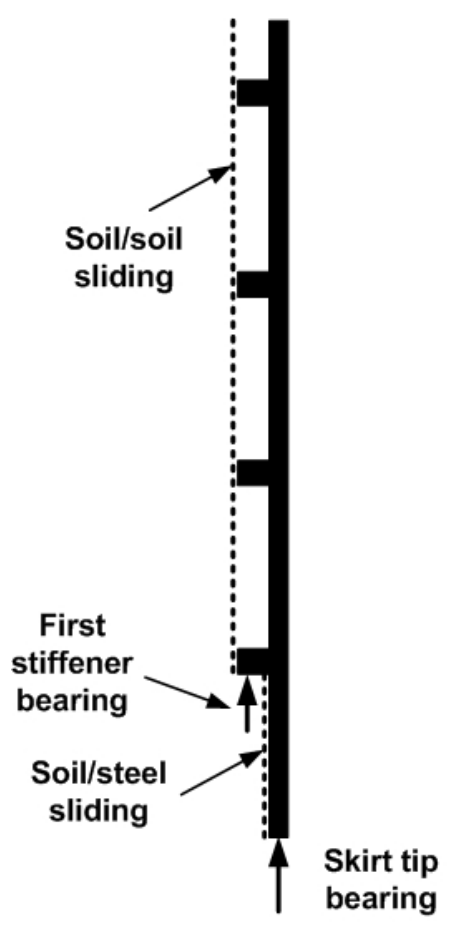

Mechanism A

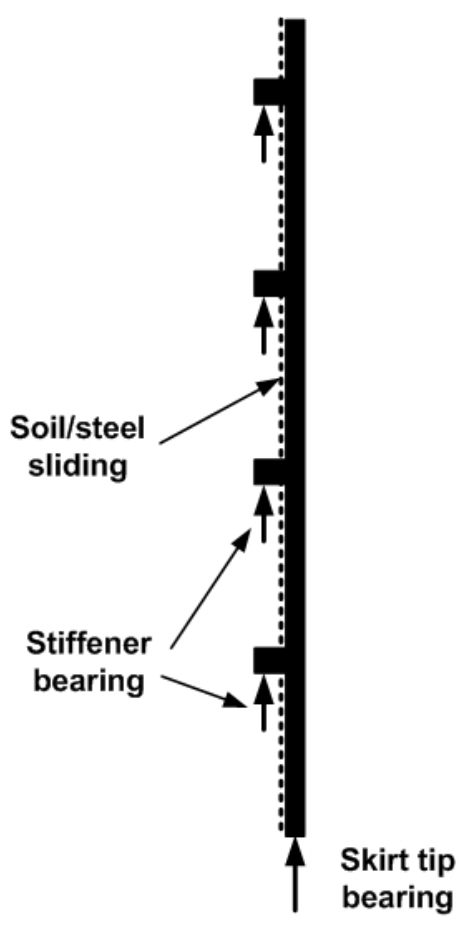

Mechanism B

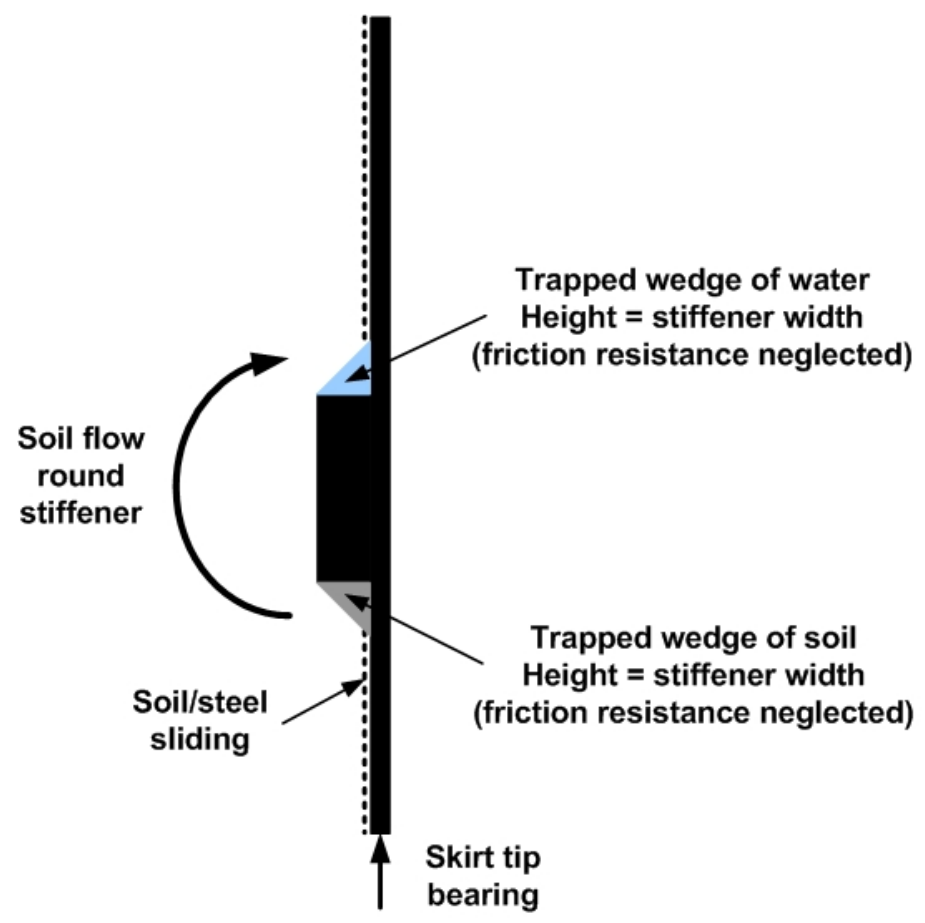

Mechanism C

Figure 2. Soil flow mechanisms for stiffened caisson installation proposed by Erbrich \& Hefer (2002) and Dendani (2003) 


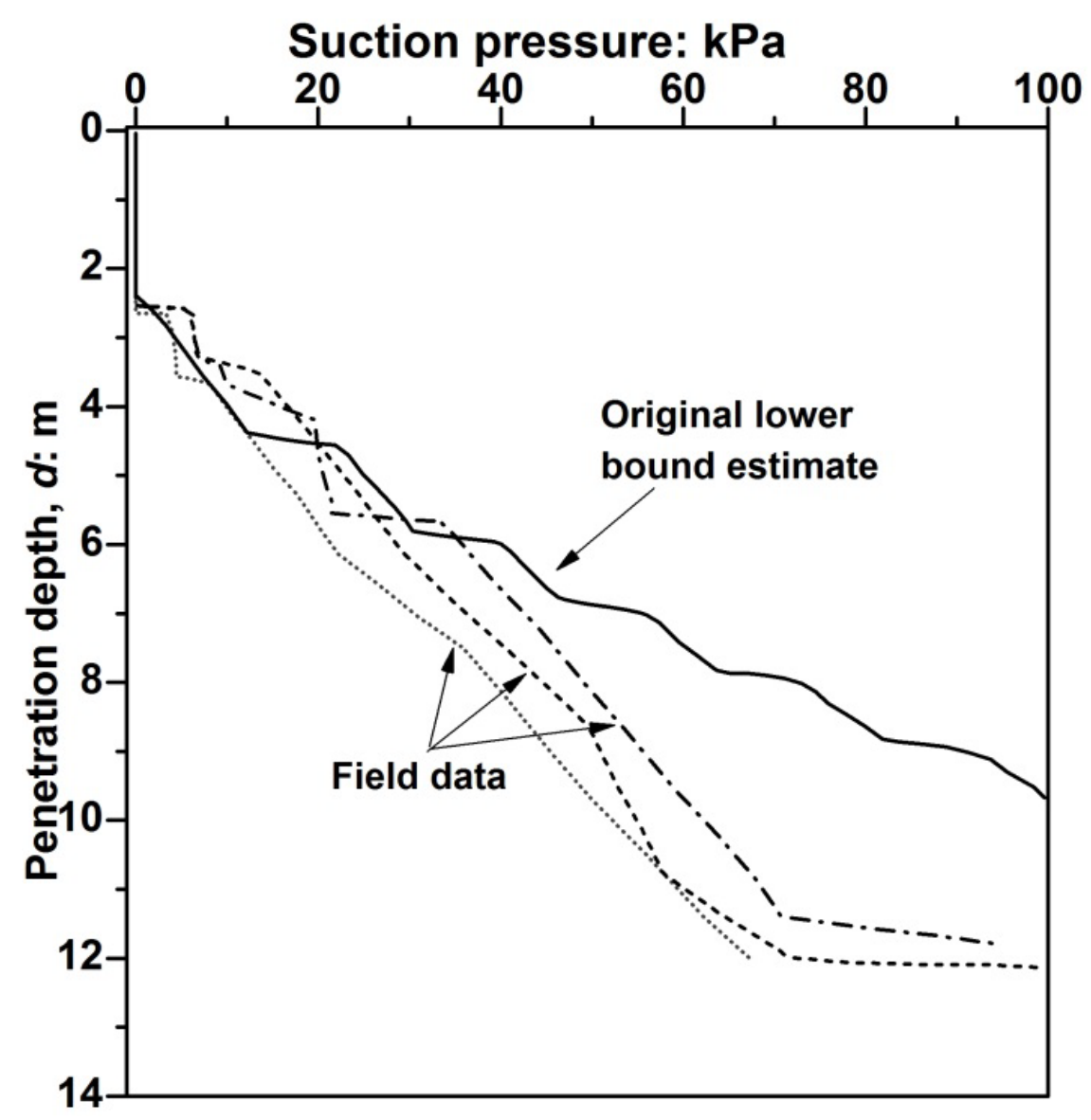

3(a) Measured vs predicted suction pressures (Laminaria: Erbrich and Hefer 2002) 


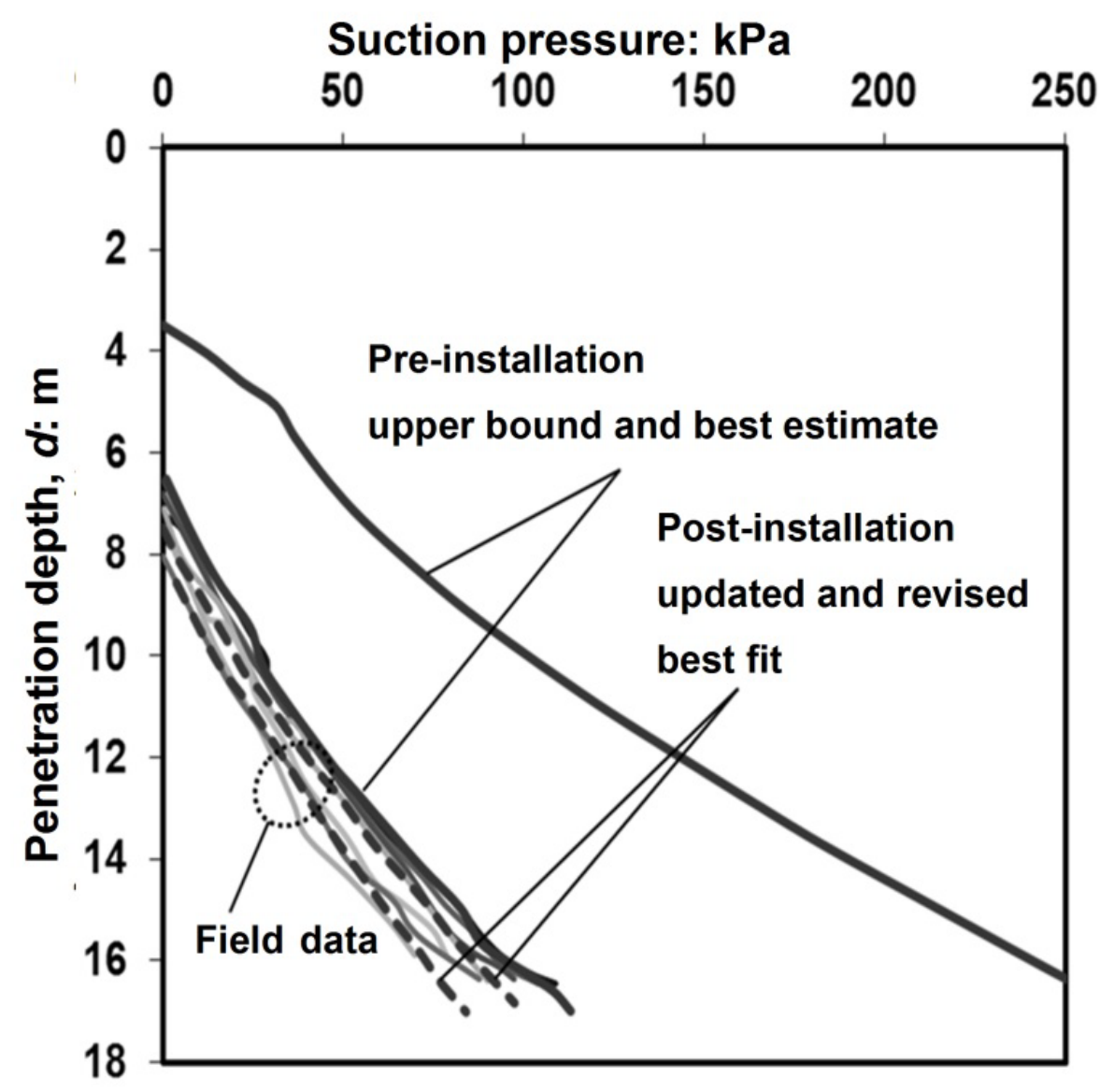

3(b) Measured vs predicted suction pressures (Girassol: Dendani and Colliat 2002;

Dendani 2003)

Figure 3. Significant overestimation related to uncertainty in soil failure mechanisms for stiffened caissons 

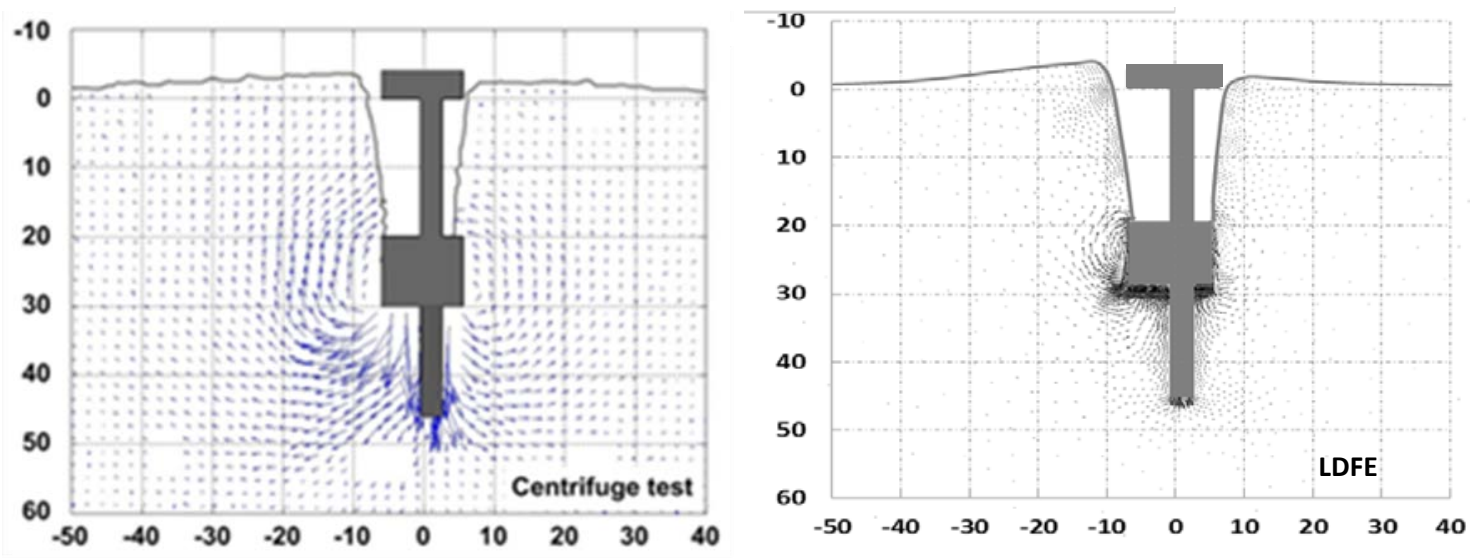

4(a) $d / t=28$ (axes in $\mathrm{mm})$
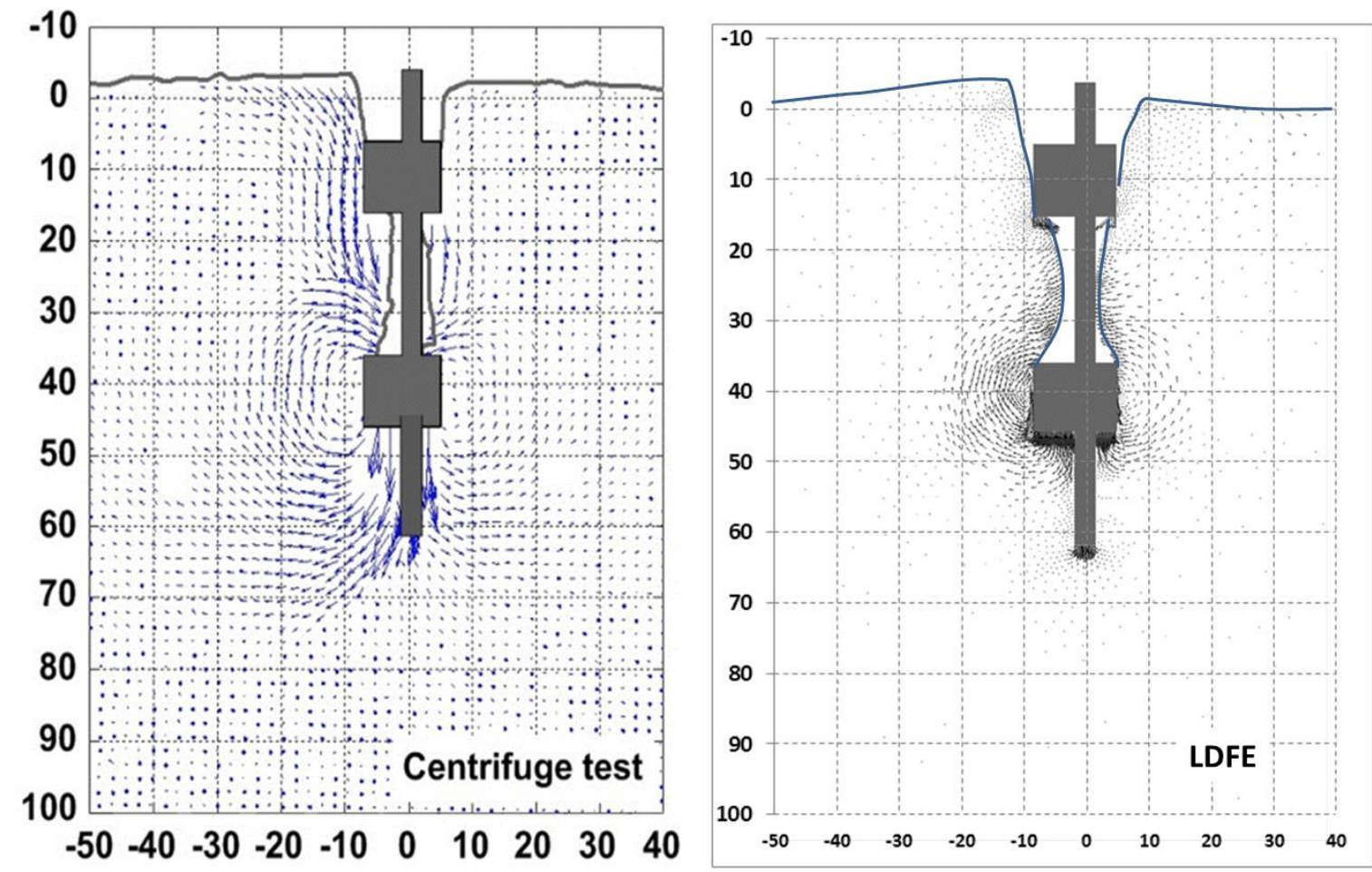

4(b) $d / t=41$ (axes in mm)

Figure 4. Comparison of soil flow mechanisms between LDFE analysis and centrifuge test data reported by Hossain et al. (2012) (plane strain, $b / t=2.0$ and 4.0, $s / h=2.0, w / b=5.3$ and 2.7, $\alpha=0.3$; Group I, Table 1) 


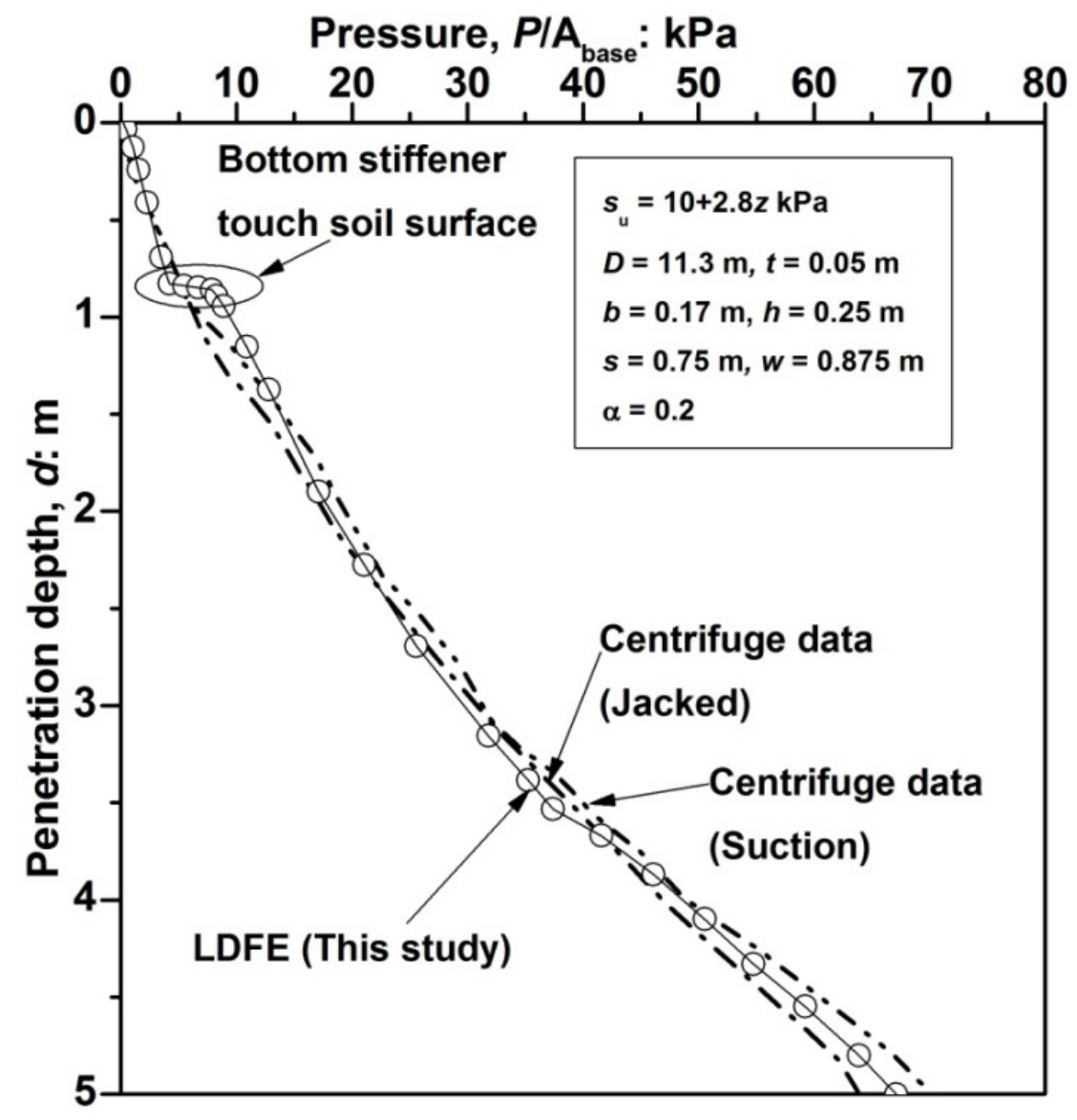

5(b) Comparison with data reported by Westgate et al. (2009) $(D / t=226, b / t=3.4$, $s / h=3.0, w / b=5.2, \alpha=0.2, k D / s_{\text {um }}=3.16, s_{\text {um }} / \gamma^{\prime} D=0.150$; Group I-B, Table 2)

Figure 5. Comparison of installation resistance between LDFE analysis and centrifuge test data 


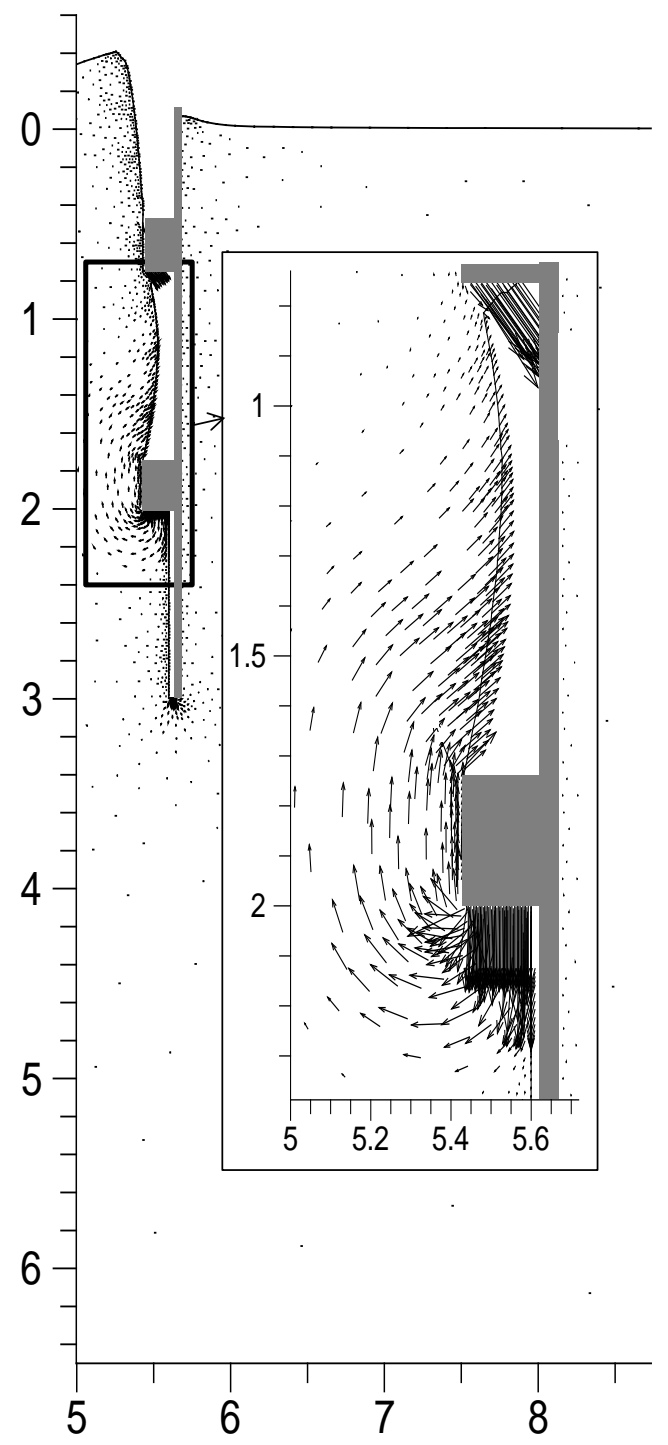

$6($ a) $d / t=60$ (axes in $\mathrm{m}$ ) 


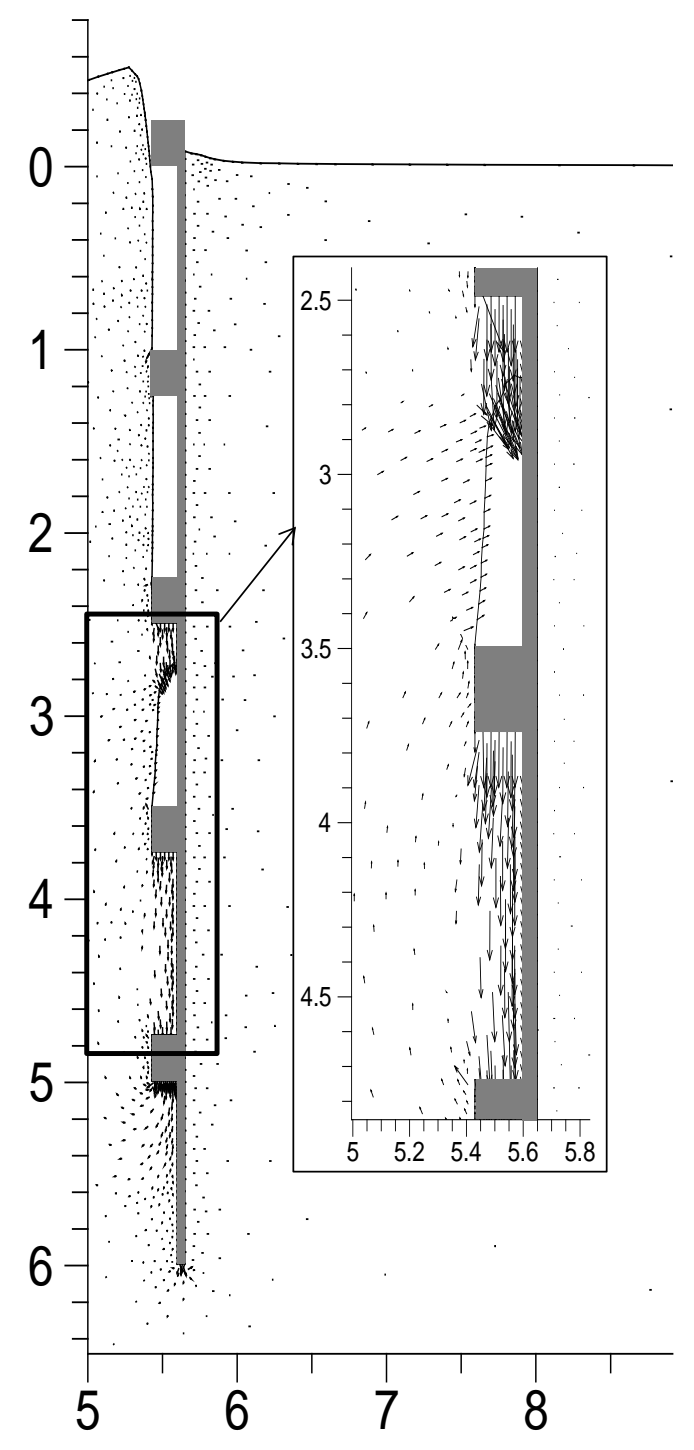

6(b) $d / t=120($ axes in $\mathrm{m})$

Figure 6. Soil flow mechanisms for stiffened caisson penetration in nonhomogeneous clay $\left(D / t=226, b / t=3.4, s / h=4.0, w / b=5.9, \alpha=0.2, k D / s_{u m}=2.26\right.$, $s_{\mathrm{um}} / \gamma^{\prime} D=0.070$; Group II, Table 1) 


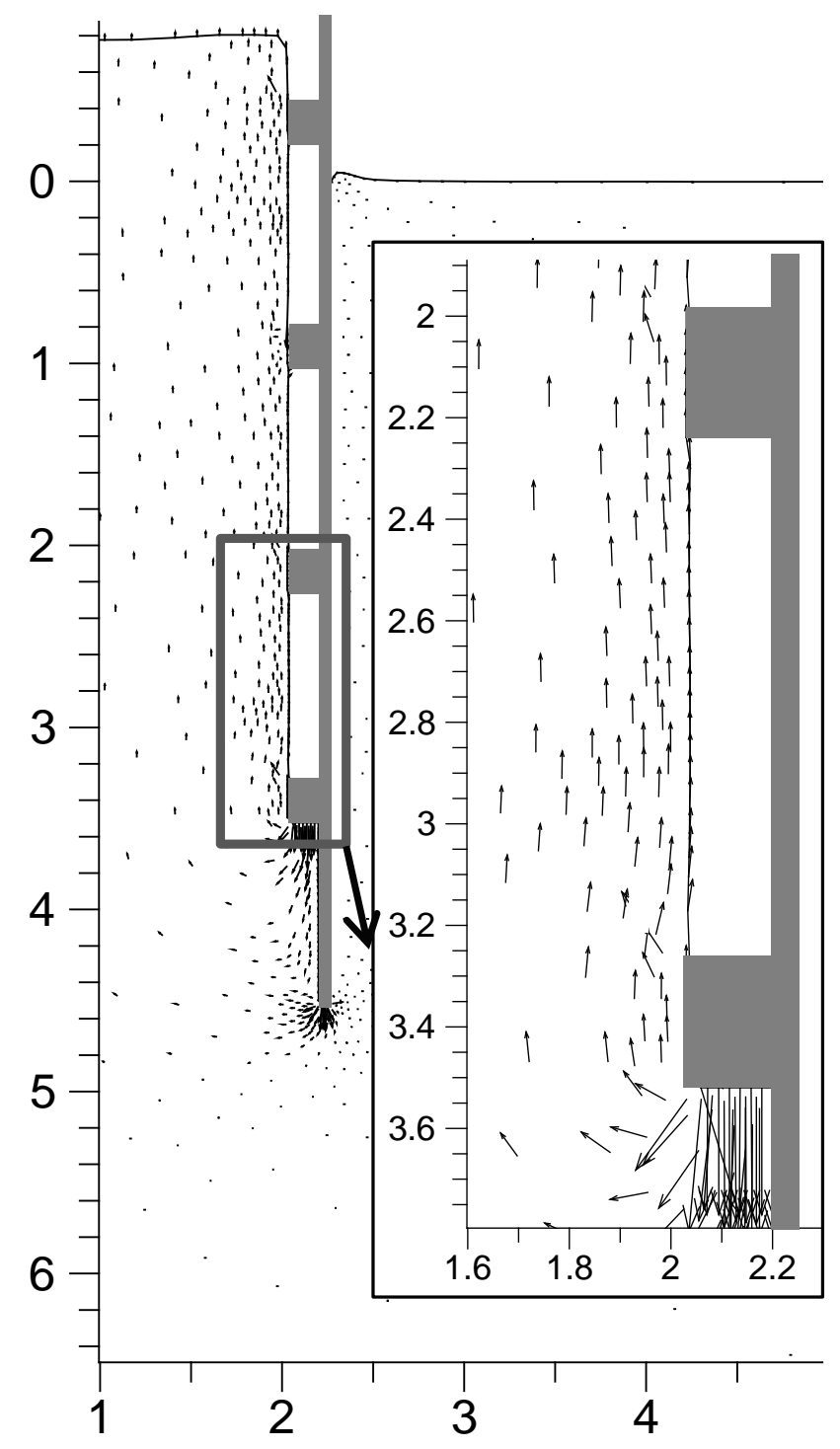

$7\left(\right.$ a) $D / t=90\left(k D / s_{\text {um }}=0.45, s_{\text {um }} / \gamma^{\prime} D=0.370\right.$; axes in $\left.\mathrm{m}\right)$ 


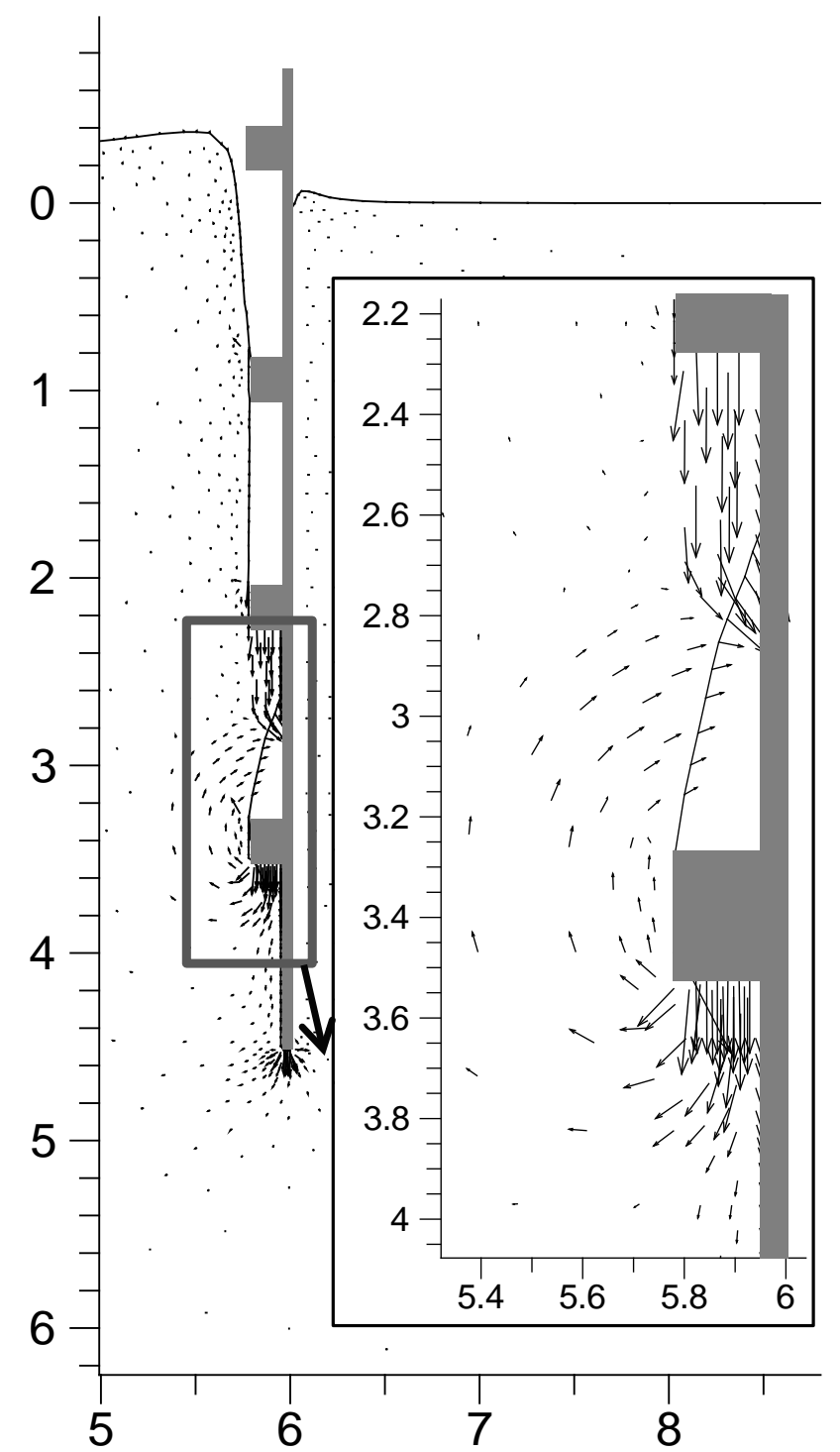

7(b) $D / t=240\left(k D / s_{u m}=1.20, s_{\text {um }} / \gamma^{\prime} D=0.140\right.$; axes in $\left.\mathrm{m}\right)$

Figure 7. Effect of caisson diameter on soil flow mechanisms at $d / t=90(b / t=3.4$, $s / h=4.0, w / b=5.9, \alpha=0.2$; Group III, Table 1) 


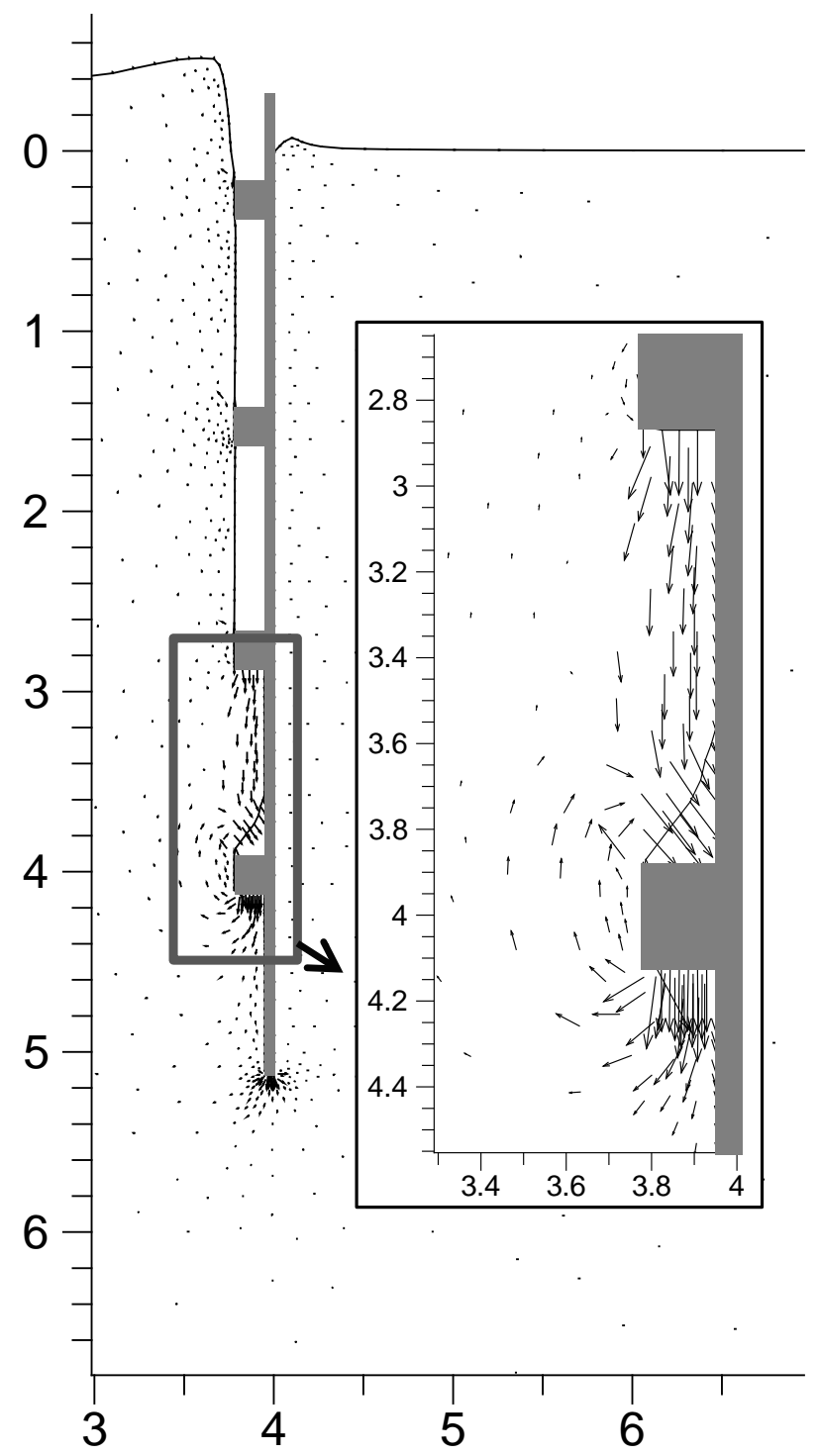

8(a) $b / t=3.4$ (axes in $m$ ) 


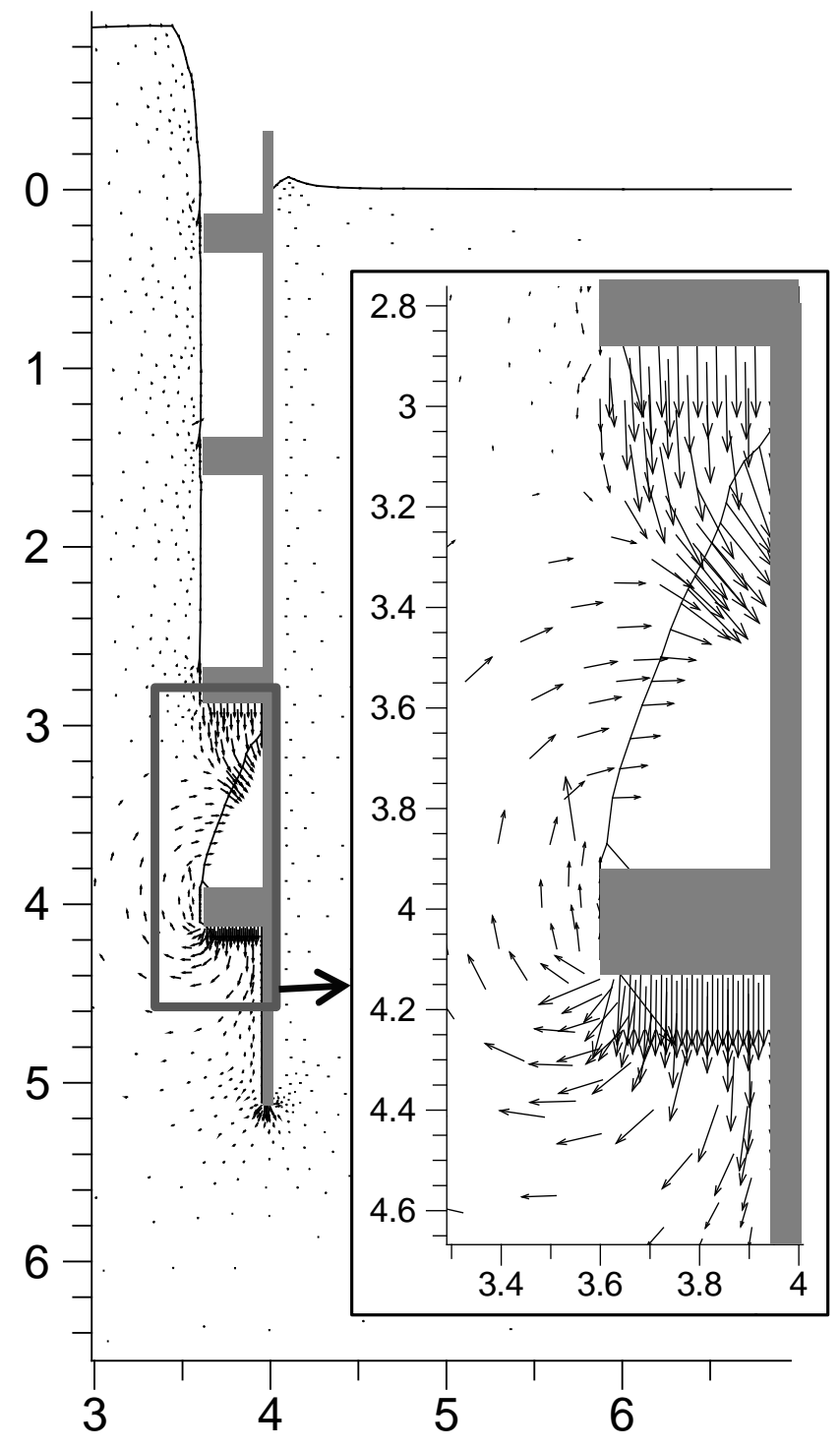

8(b) $b / t=7.0$ (axes in $\mathrm{m}$ )

Figure 8. Effect of stiffener width on soil flow mechanisms at $d / t=102(D / t=160$, $s / h=4.0, \alpha=0.2, k D / s_{\mathrm{um}}=0.80, s_{\mathrm{um}} / \gamma^{\prime} D=0.210$; Group IV, Table 1) 


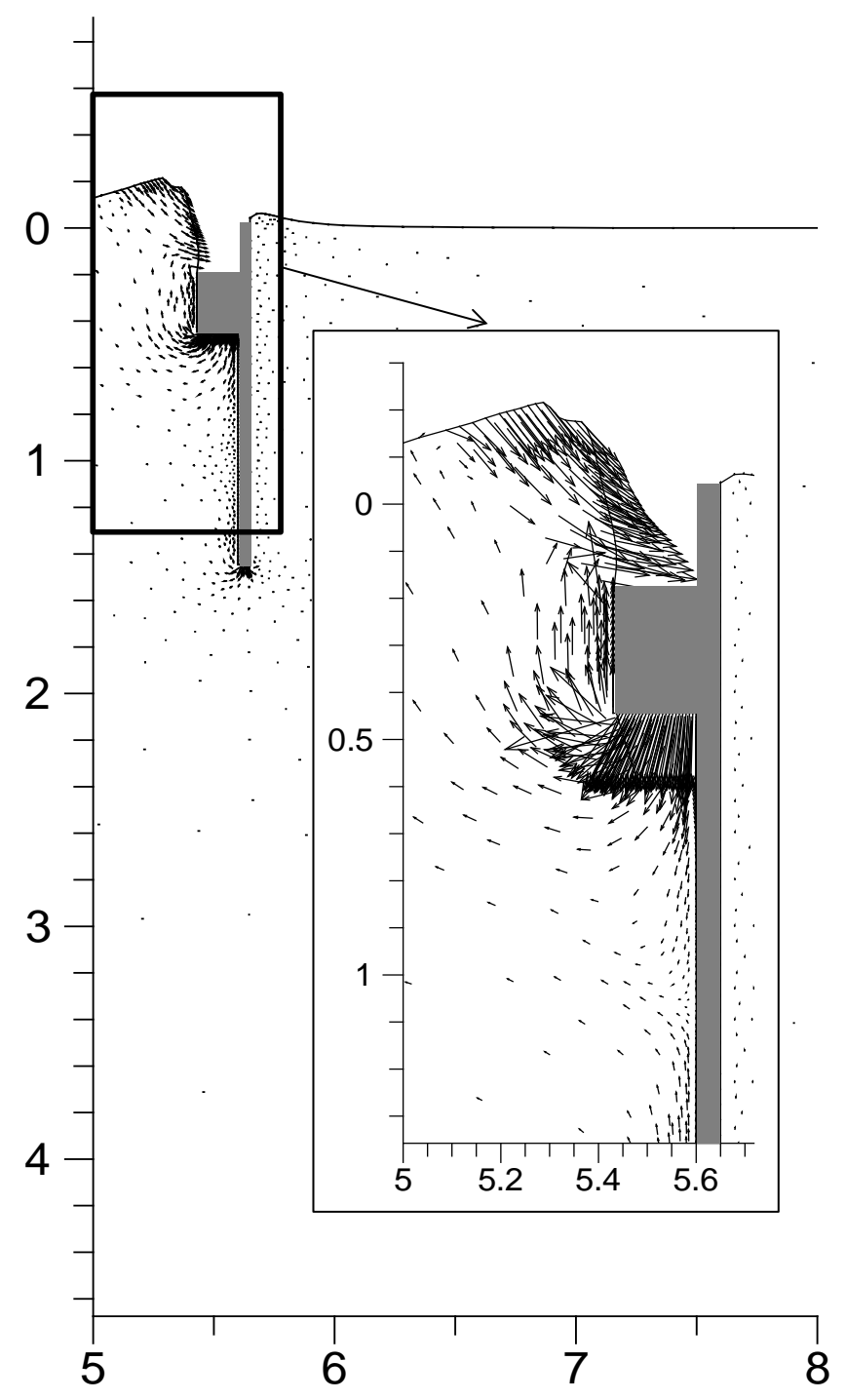

9 (a) $d / t=29$ (axes in $\mathrm{m}$ ) 


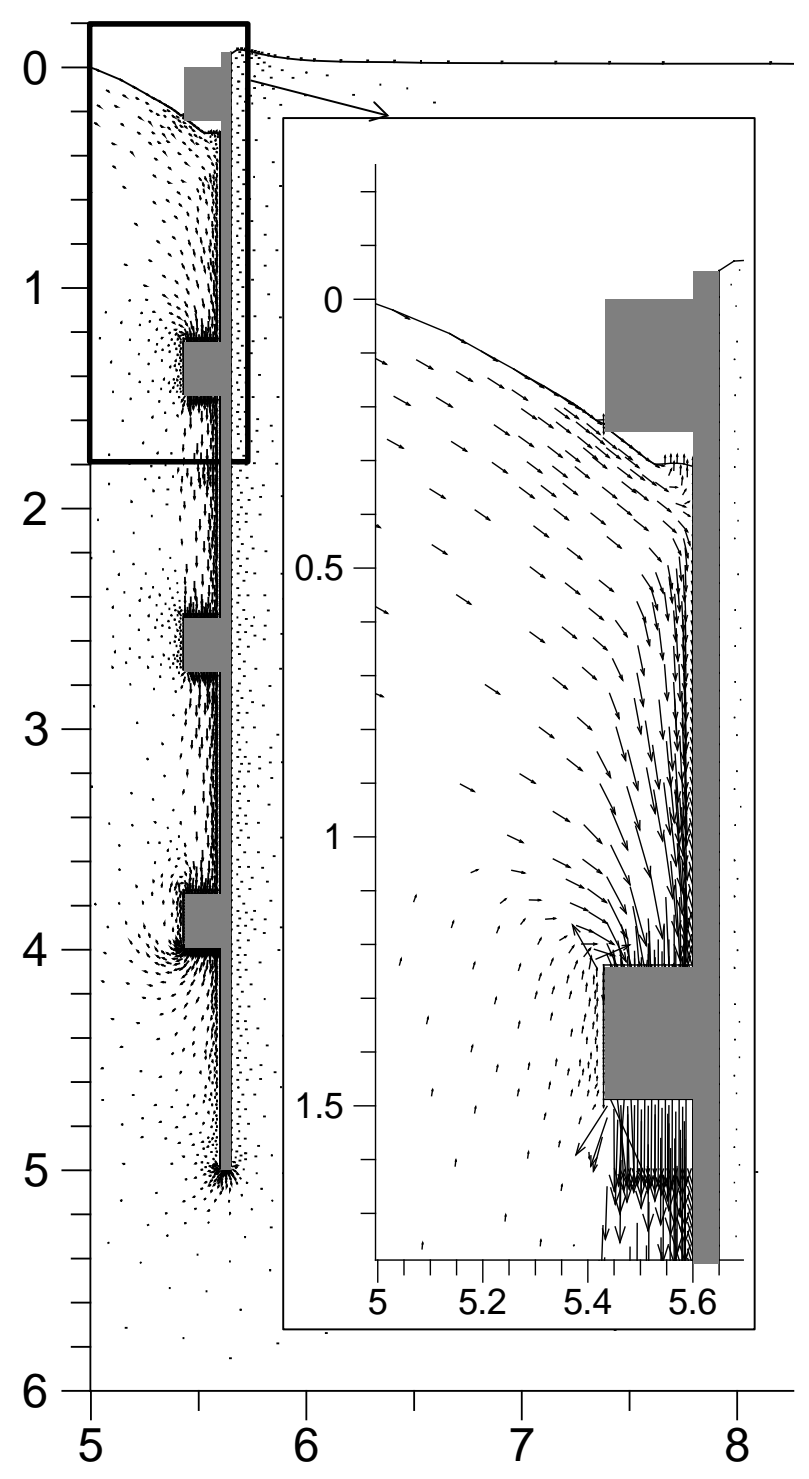

9(b) $d / t=100$ (axes in $\mathrm{m}$ )

Figure 9. Effect of soil strength non-homogeneity and normalized strength on soil flow mechanisms $\left(k D / s_{\mathrm{um}}=22.60, s_{\mathrm{um}} / \gamma^{\prime} D=0.007, D / t=226, b / t=3.4, s / h=\right.$ 4.0, $w / b=5.2, \alpha=0.2$; Group V, Table 1) 


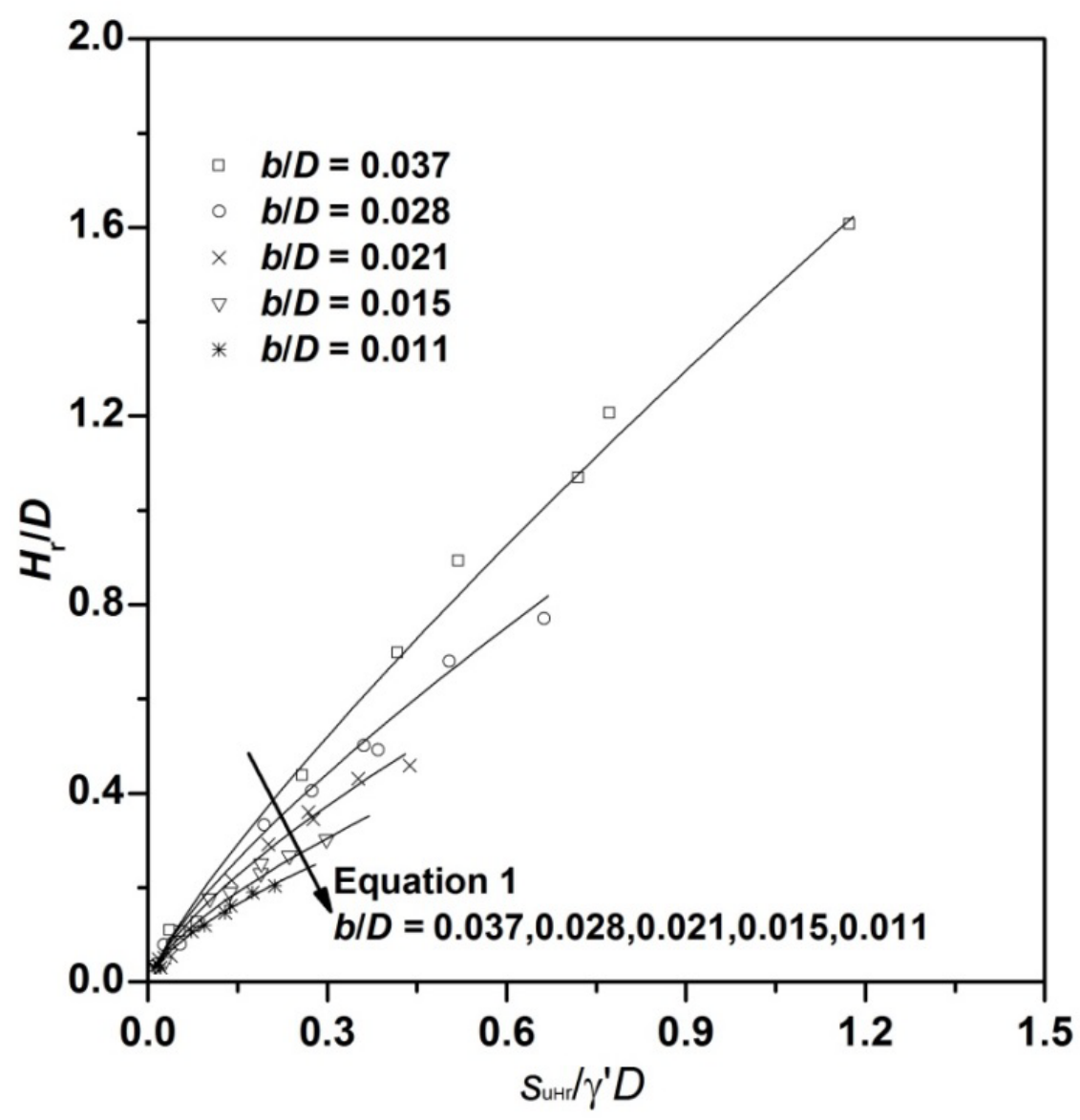

10(a) Depth of rotational flow $\left(H_{\mathrm{r}}\right)$ 


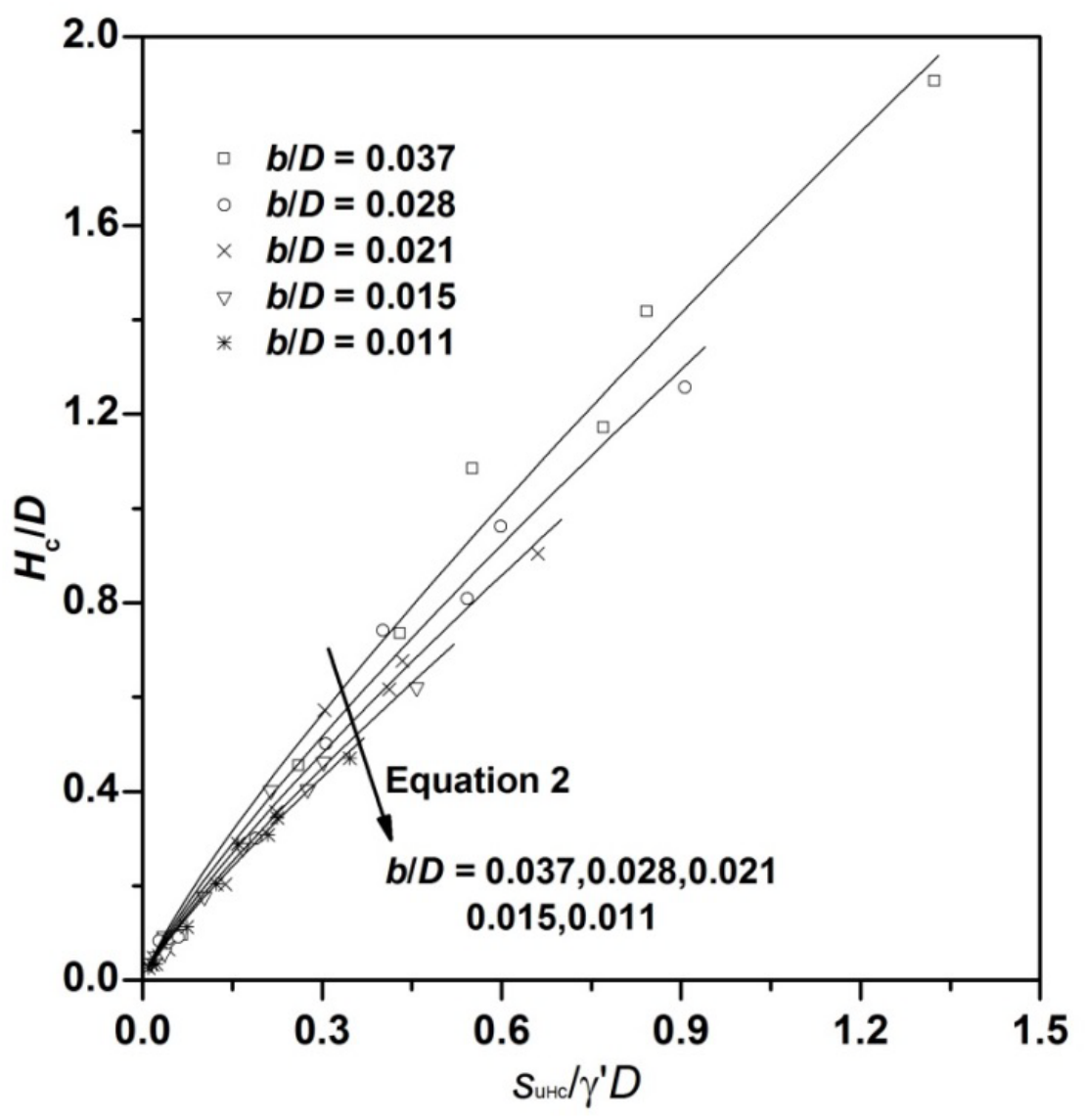

10(b) Limiting cavity depth $\left(H_{\mathrm{c}}\right)$

Figure 10. Design charts for estimating critical depths of soil infill in gaps between embedded stiffeners (Group VI, Table 1) 


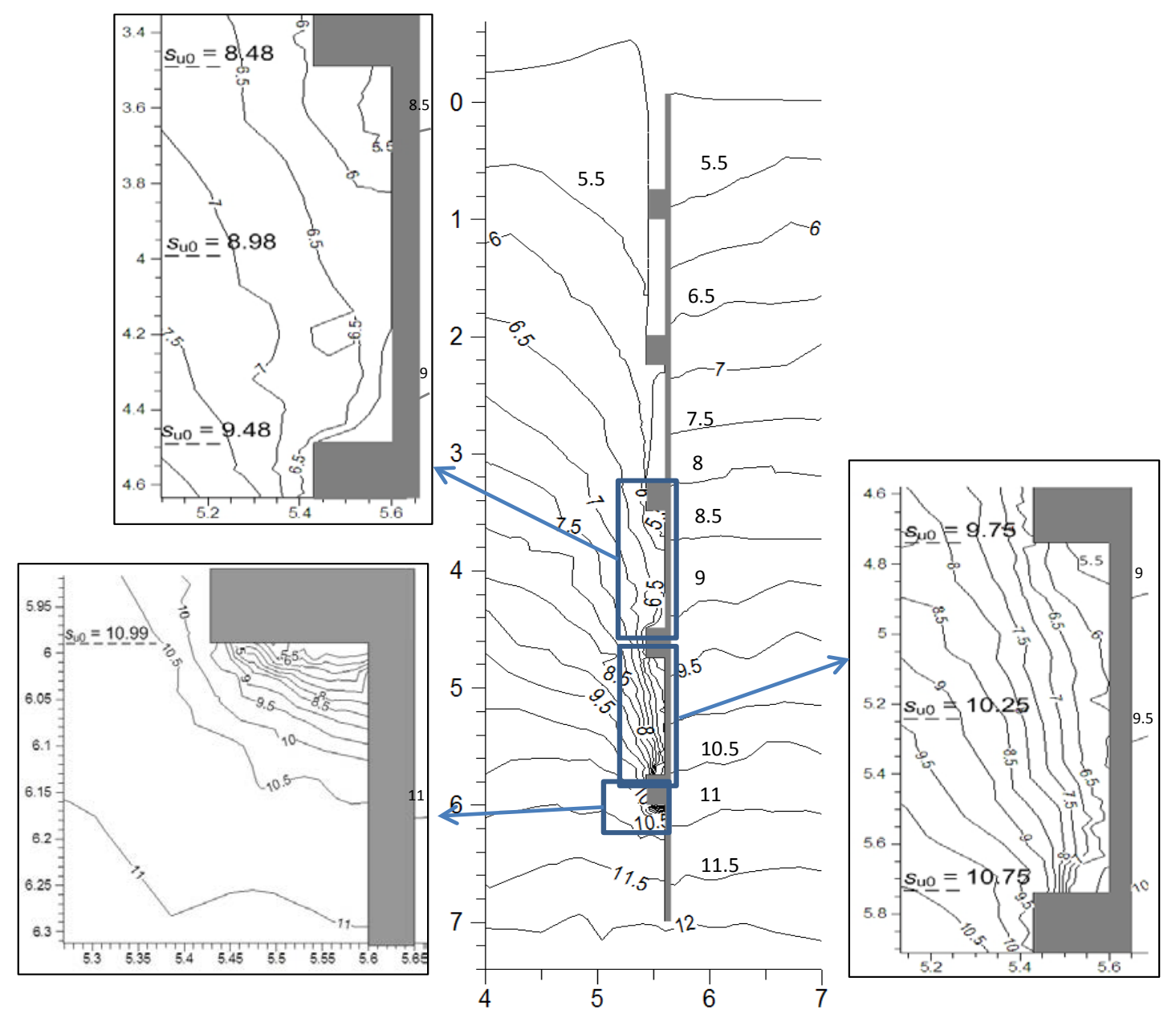

11(a) Case A: $d / t=140, D / t=226, b / t=3.4, s / h=4.0, w / b=5.2, \alpha=0.2, s_{\mathrm{u}}=5+1 z$ $\mathrm{kPa}$ (in Group VI, Table 1) 


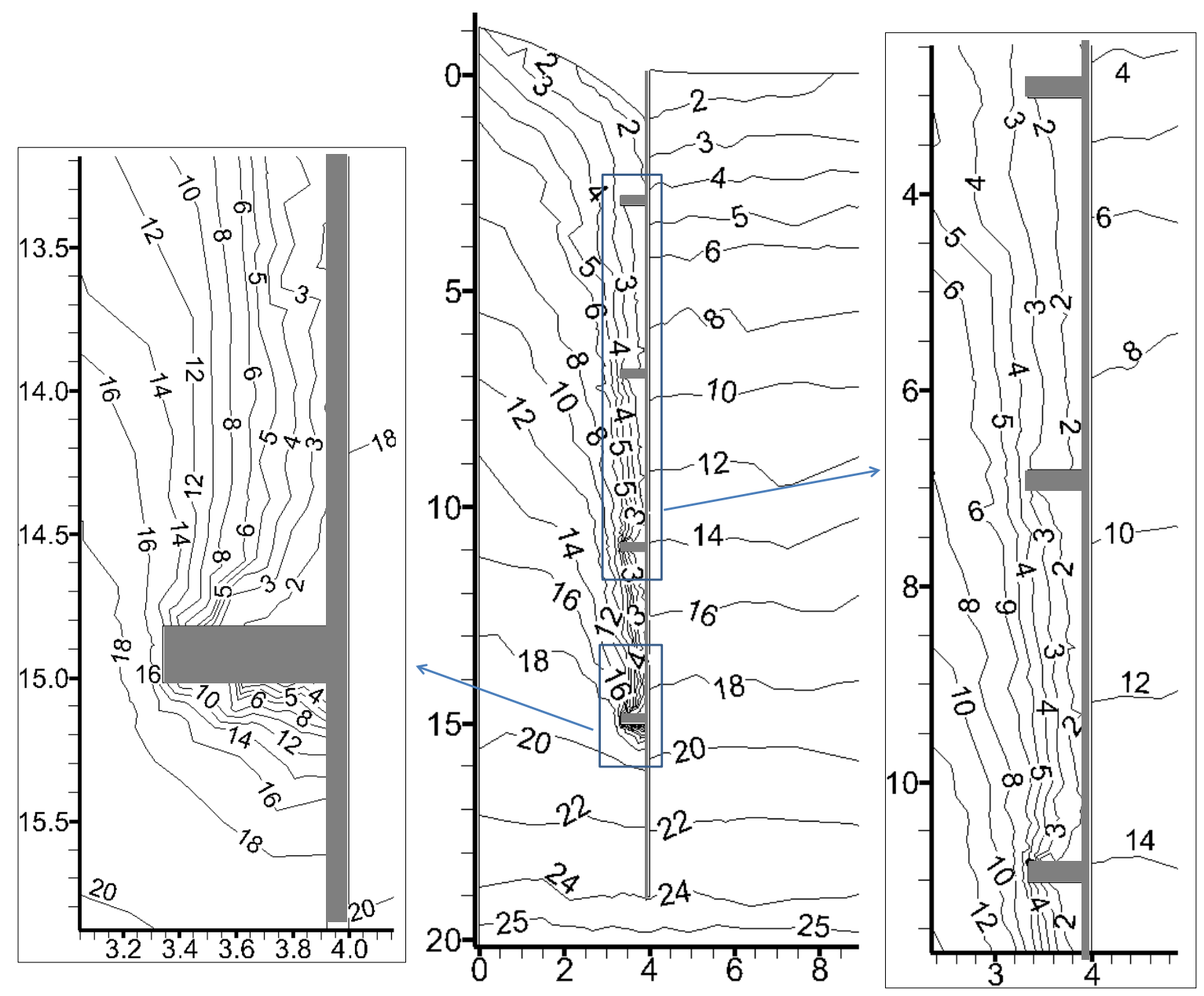

11(b) Case B: $d / t=238, D / t=100, b / t=7.3, s / h=19.0, w / b=8.0, \alpha=0.3, s_{\mathrm{u}}=1.33+$ $1.2 \mathrm{z} \mathrm{kPa}$ (Group I-A, Table 1)

Figure 11. Strength contours for stiffened caisson penetration in nonhomogeneous clay 


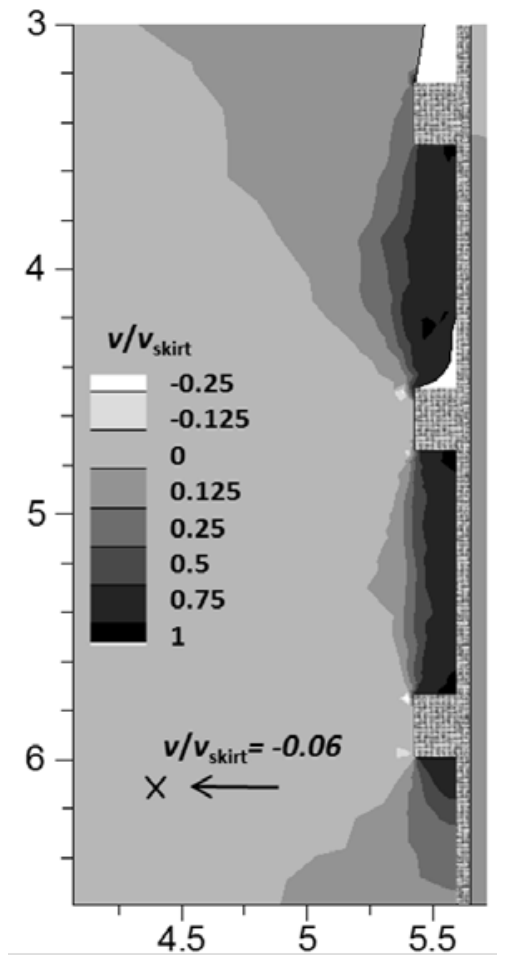

Case A

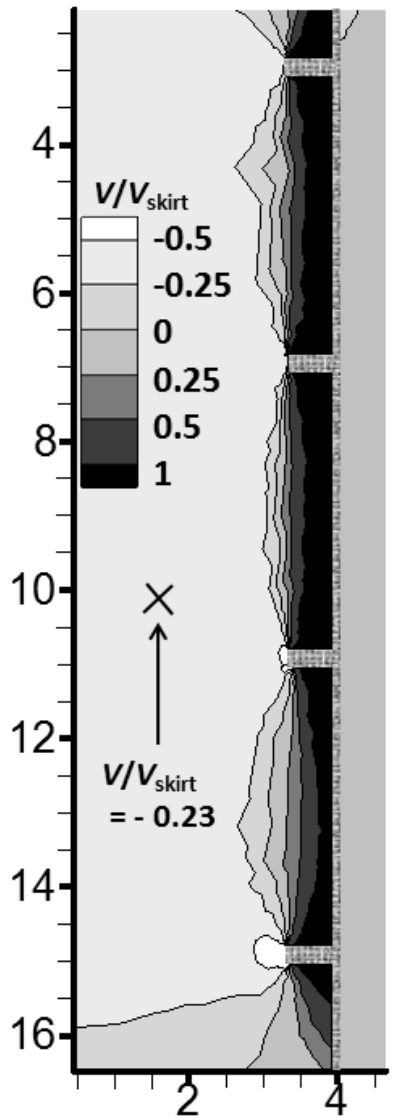

Case B

Figure 12. Soil vertical flow velocity contours for stiffened caisson penetration in clay 


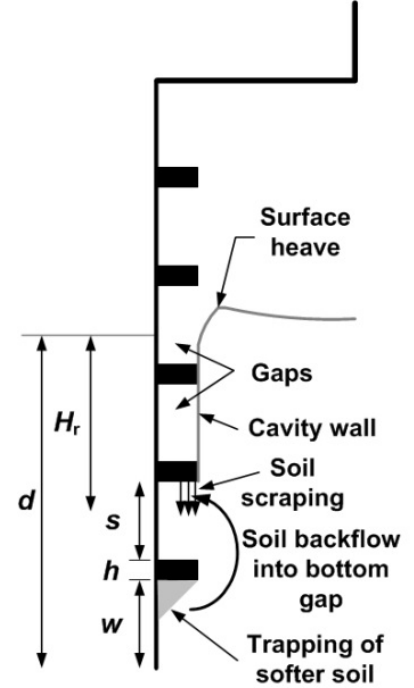

$(d-w)>H_{r}$

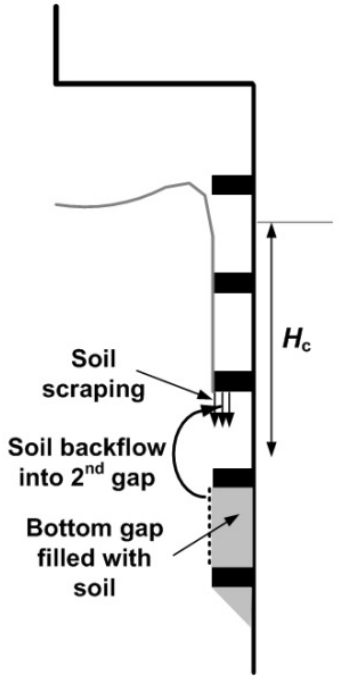

(3a) End bearing

(1) Outer friction $\left(F_{0}\right)$ bottom stiffener $\left(F_{\text {iabb }}\right)$

(3b) End bearing at base of bottom stiffener $\left(F_{t}\right)$

(2a) Inner skirt wall friction below bottom stiffener $\left(F_{i b}\right)$ at tip of skirt $\left(F_{\mathrm{b}}\right)$

New mechanism

Figure 13. A new mechanism for stiffened caisson installation in clay 


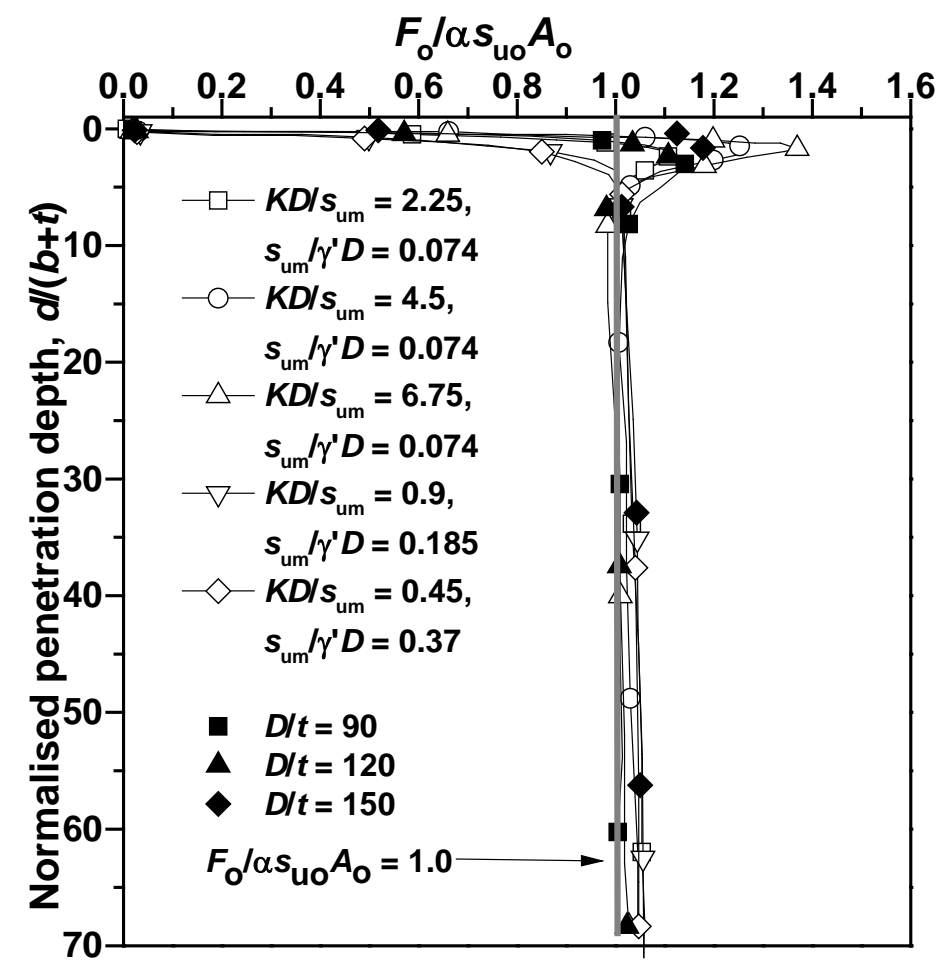

14(a) Effect of normalized caisson diameter and of soil strength non-homogeneity , $D / t\left(b / t=3.4, s / h=12.0, w / b=11.8, \alpha=0.2, k D / s_{\text {um }}=2.25 \sim 3.75, s_{\text {um }} / \gamma^{\prime} D=\right.$ 0.074 0.044; Group II-A, Table 2) $k D / s_{\text {um }}$, and normalized strength, $s_{\mathrm{um}} / \gamma^{\prime} D(D / t=$ $90, b / t=3.4, s / h=12.0, w / b=11.8, \alpha=0.2$; Group II-B, Table 2)

Figure 14. Friction along outer periphery of caisson $\left(F_{0}\right)$ 


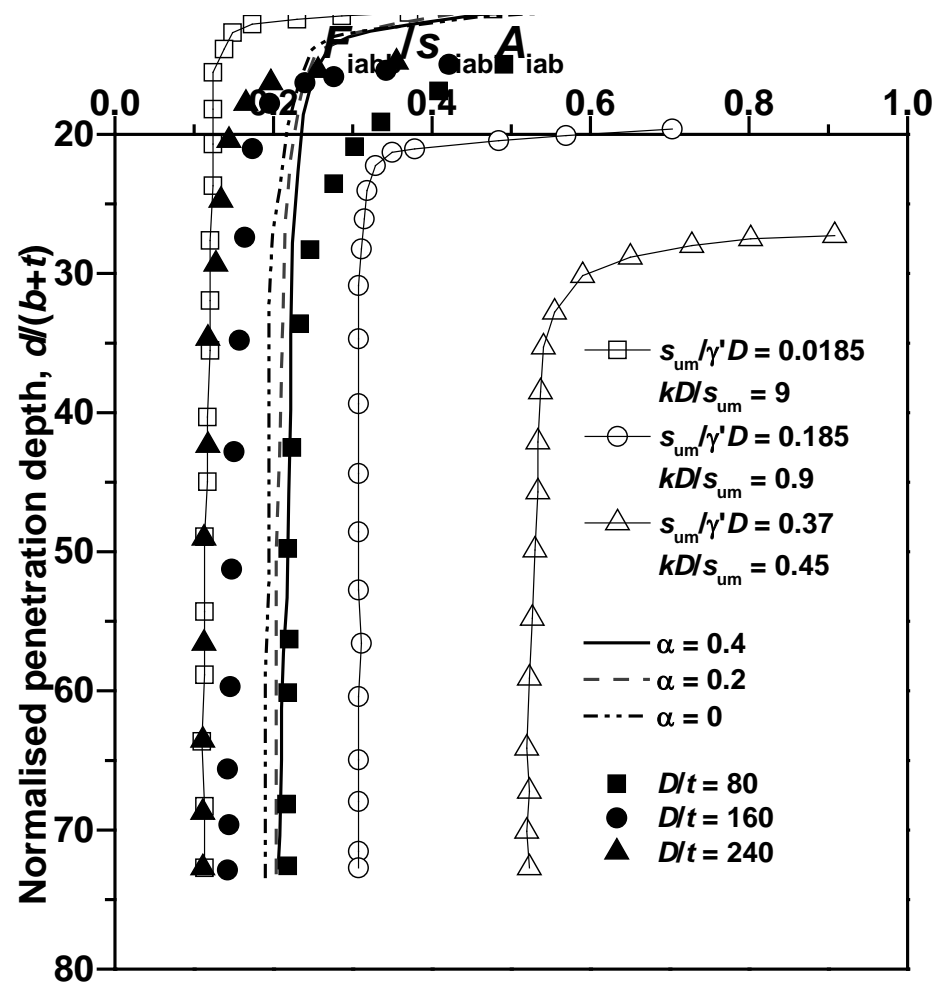

15(a) Effect of coefficient of friction, diameter and normalized strength , $\alpha$ ( $D / t=90$, $b / t=3.4, s / h=12.0, w / b=11.8, k D / s_{\text {um }}=2.25, s_{\mathrm{um}} / \gamma^{\prime} D=0.074$; Group III-A, Table 2) ; D/t $\left(b / t=3.4, s / h=12.0, w / b=11.8, \alpha=0.2, k D / s_{\mathrm{um}}=2.00 \sim 6.00, s_{\mathrm{um}} / \gamma^{\prime} D=\right.$ 0.083 0.028; Group III-B, Table 2); kD/sum, and normalized strength, $s_{\mathrm{um}} / \gamma^{\prime} D(D / t$ $=90, b / t=3.4, s / h=4.0, w / b=5.9, \alpha=0.2$; Group III-C, Table 2) 


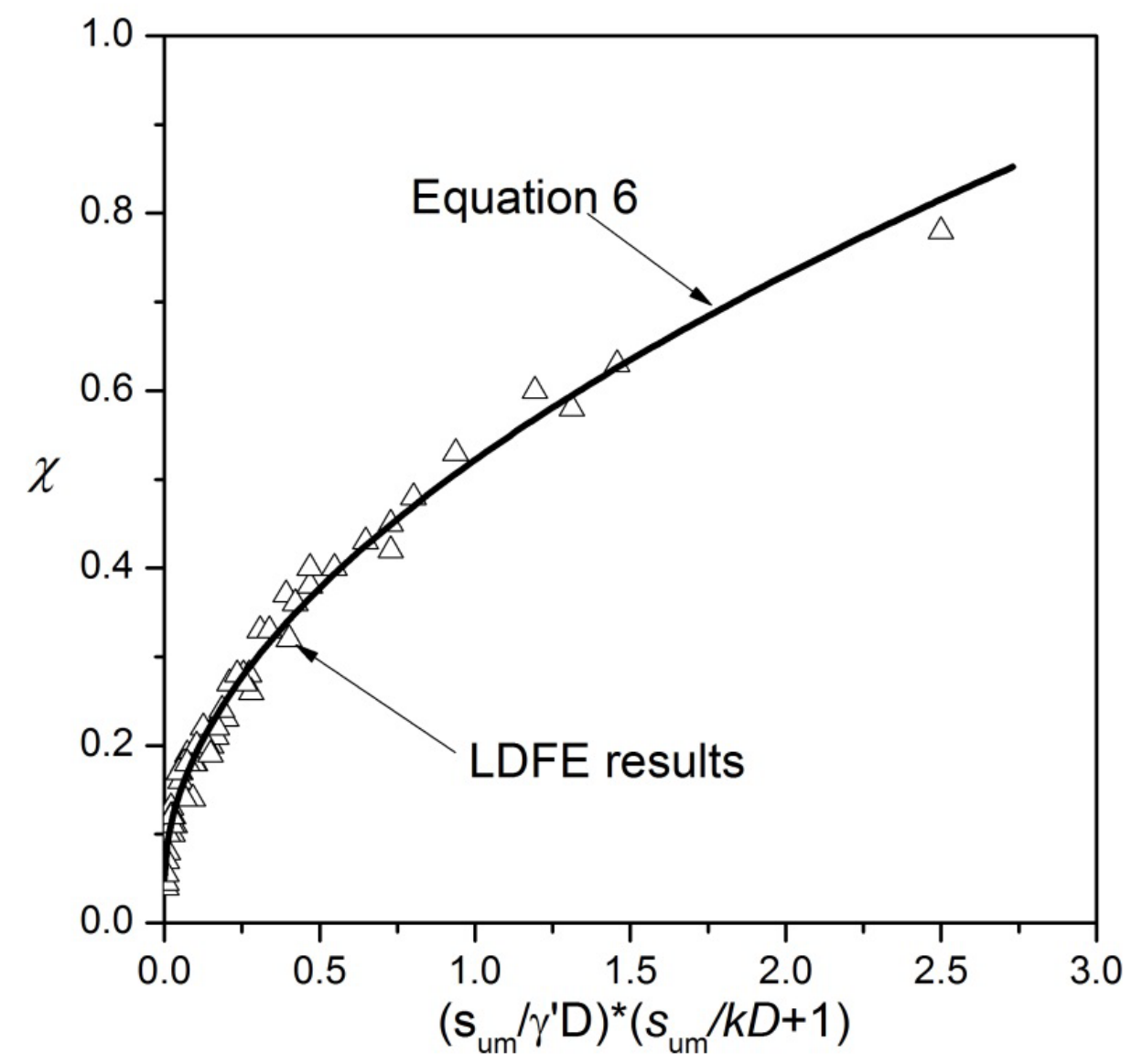

15(b) Design chart for coefficient of inner friction above bottom stiffener, $\chi(D / t=$ $80 \sim 300, b / t=3.4, s / h=12.0, w / b=11.8, \alpha=0.2$; Group III-D, Table 2)

Figure 15. Inner friction above bottom stiffener $\left(F_{\text {iabb }}\right)$ 


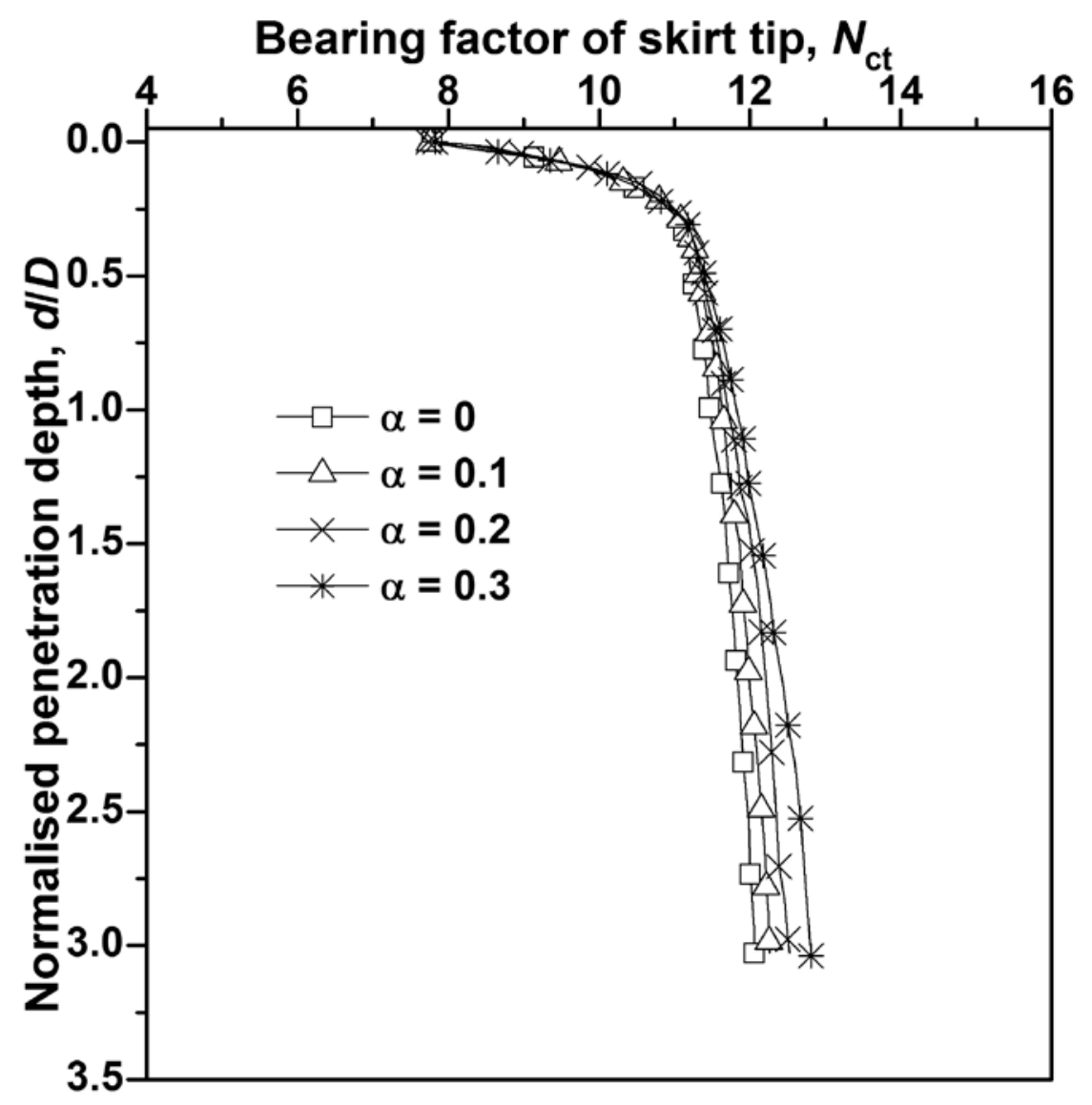

16(a) Effect of coefficient of friction, $\alpha(D / t=90, b / t=3.4, s / h=12.0, w / b=11.8$, $k D / s_{\text {um }}=0.90, s_{\mathrm{um}} / \gamma^{\prime} D=0.185$; in Group IV, Table 2) 


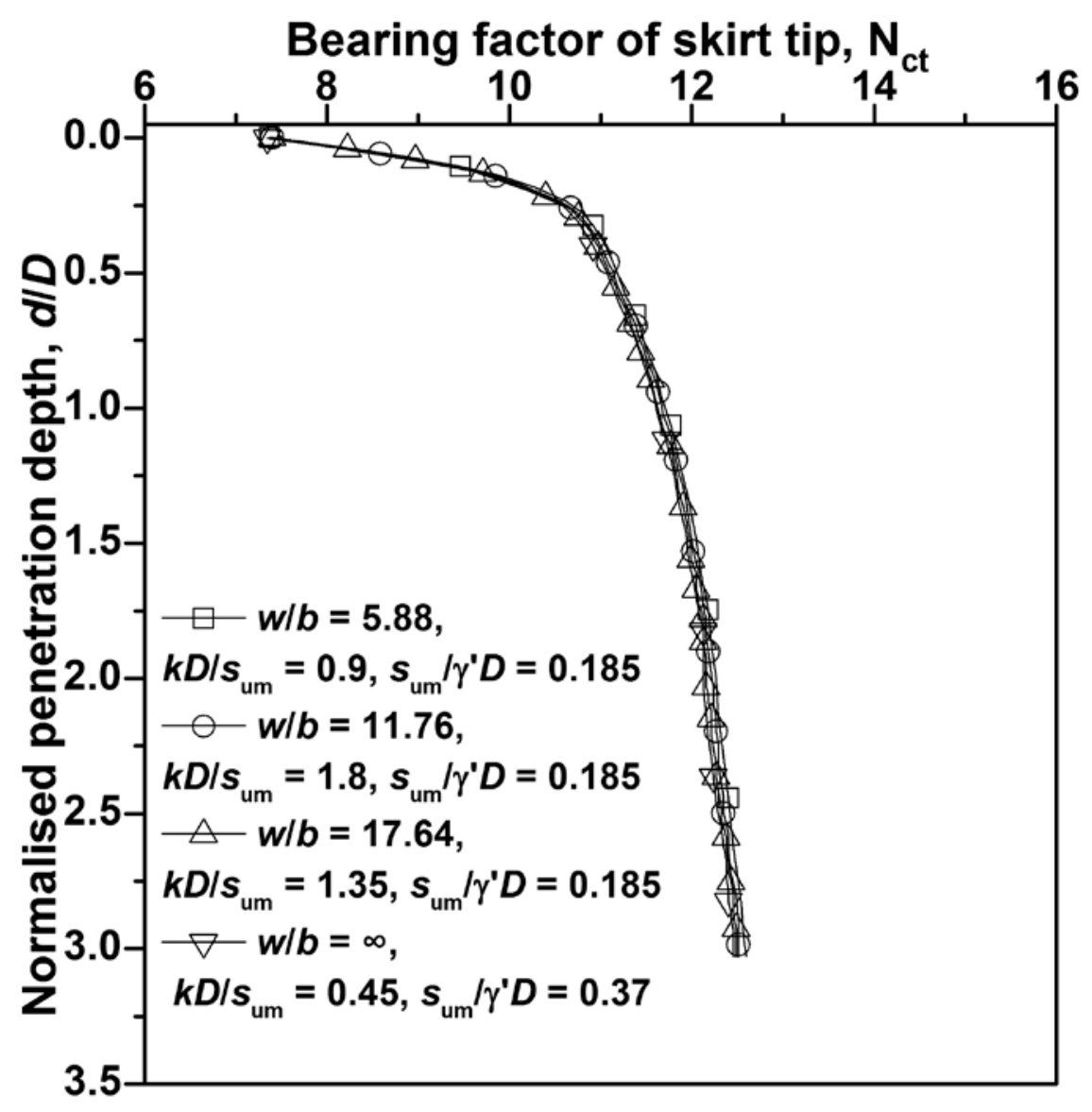

16(b) Effect of distance from bottom stiffener to skirt tip, $w / b$, and soil strength nonhomogeneity, $k D / s_{\mathrm{um}}$, and normalized strength, $s_{\mathrm{um}} / \gamma^{\prime} D(D / t=90, b / t=3.4, s / h=12.0$, $\alpha=0.2$; in Group IV, Table 2) 


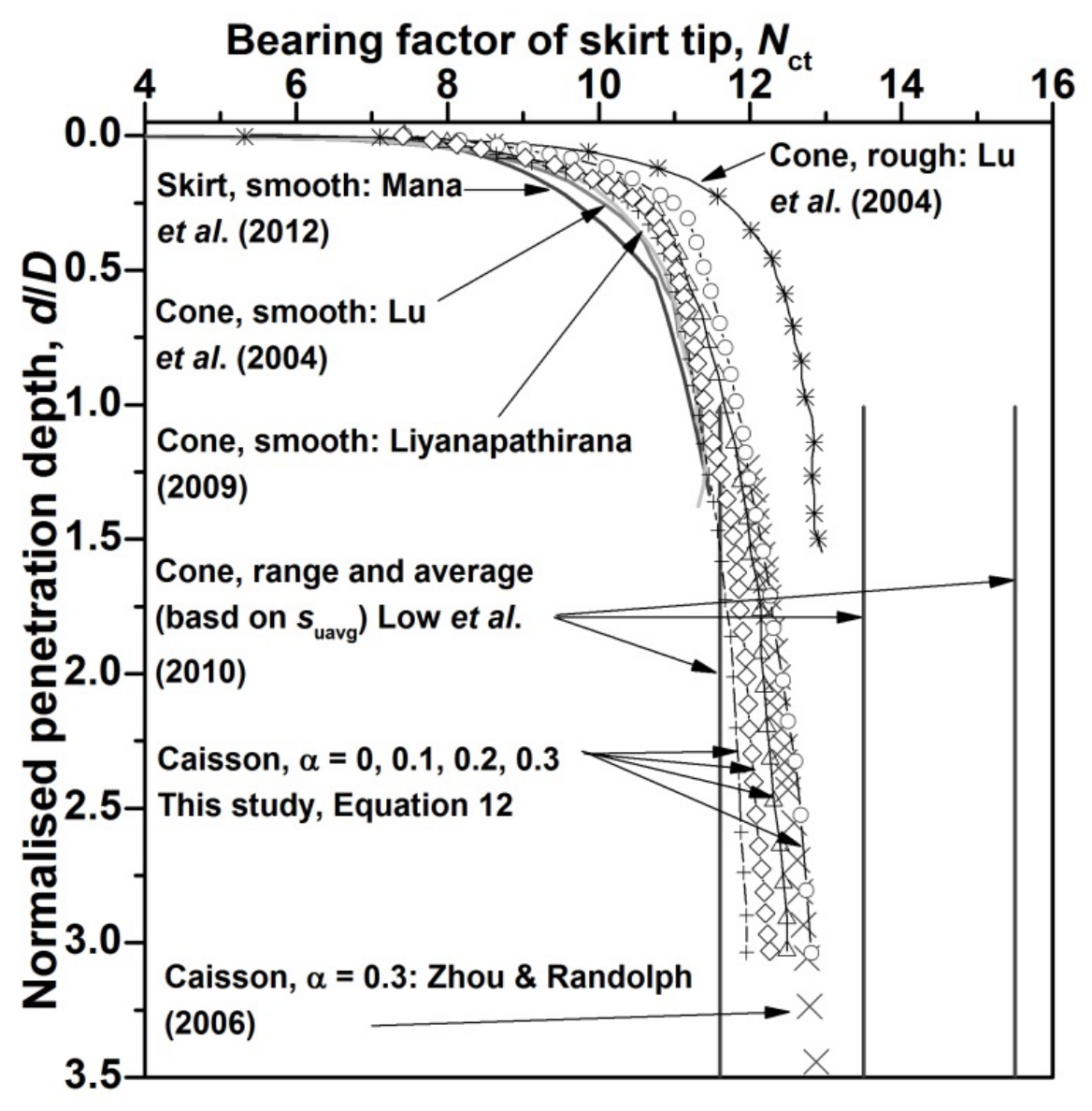

16(c) Design chart for $N_{\mathrm{ct}}$

Figure 16. End bearing factor at skirt tip $\left(N_{\mathrm{ct}}\right)$ 


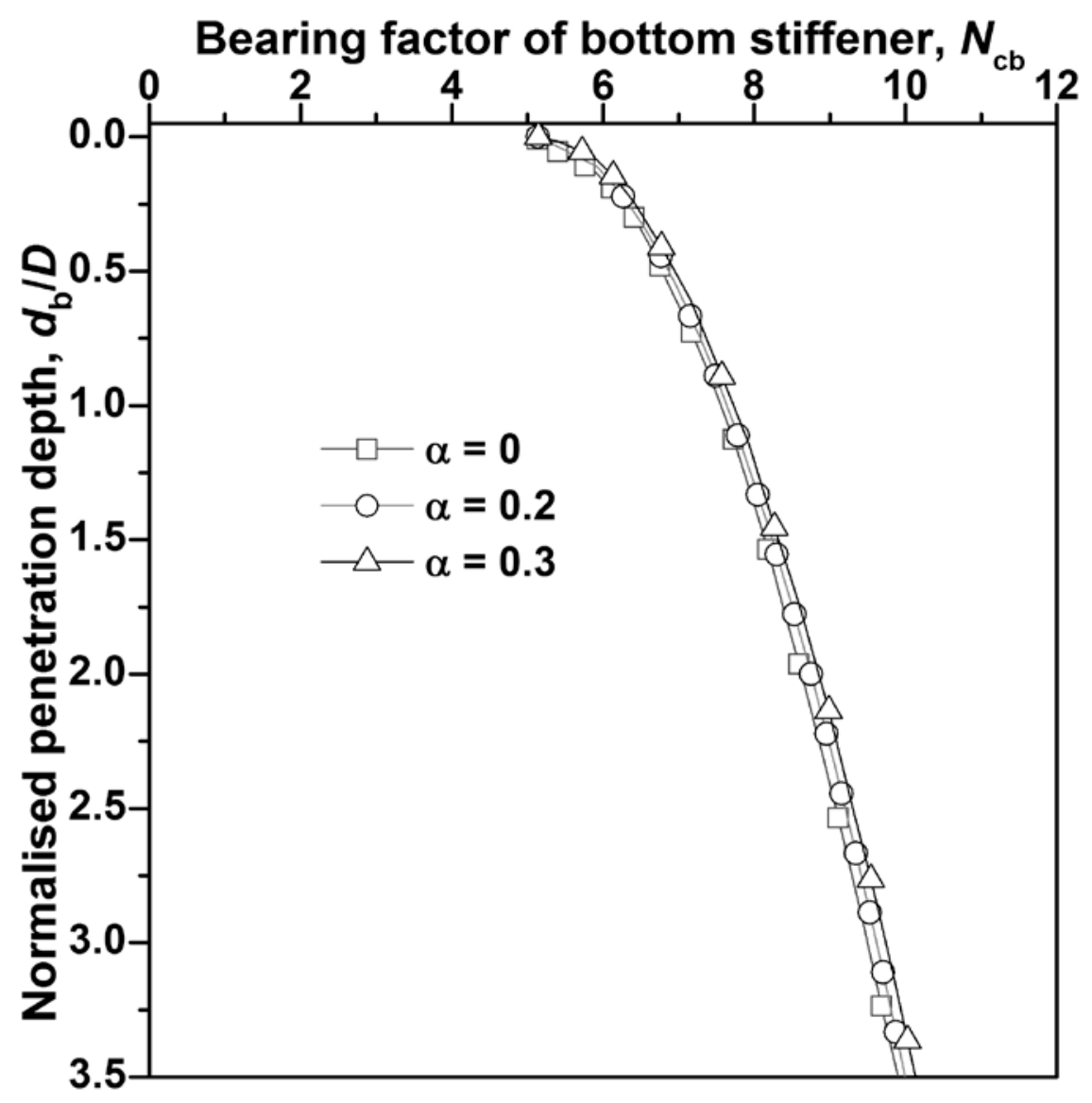

17(a) Effect of coefficient of friction, $\alpha(D / t=90, b / t=3.4, s / h=12.0, w / b=11.8$, $k D / s_{\mathrm{um}}=0.90, s_{\mathrm{um}} / \gamma^{\prime} D=0.185$; in Group IV, Table 2) 


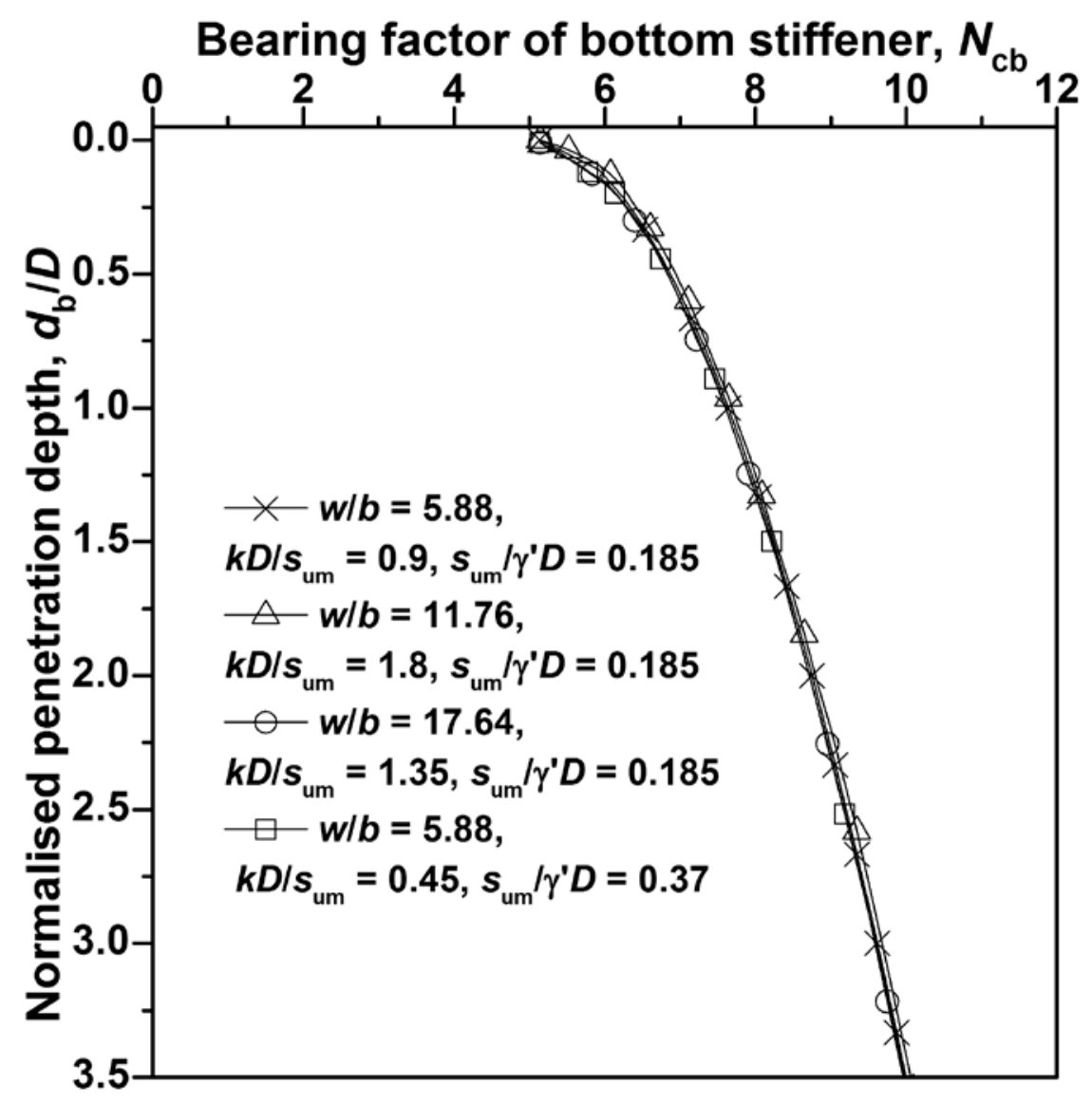

17(b) Effect of distance from bottom stiffener to skirt tip, $w / b$, and soil strength nonhomogeneity, $k D / s_{\mathrm{um}}$, and normalized strength, $s_{\mathrm{um}} / \gamma^{\prime} D(D / t=90, b / t=3.4, s / h=12.0$, $\alpha=0.2$; in Group IV, Table 2) 


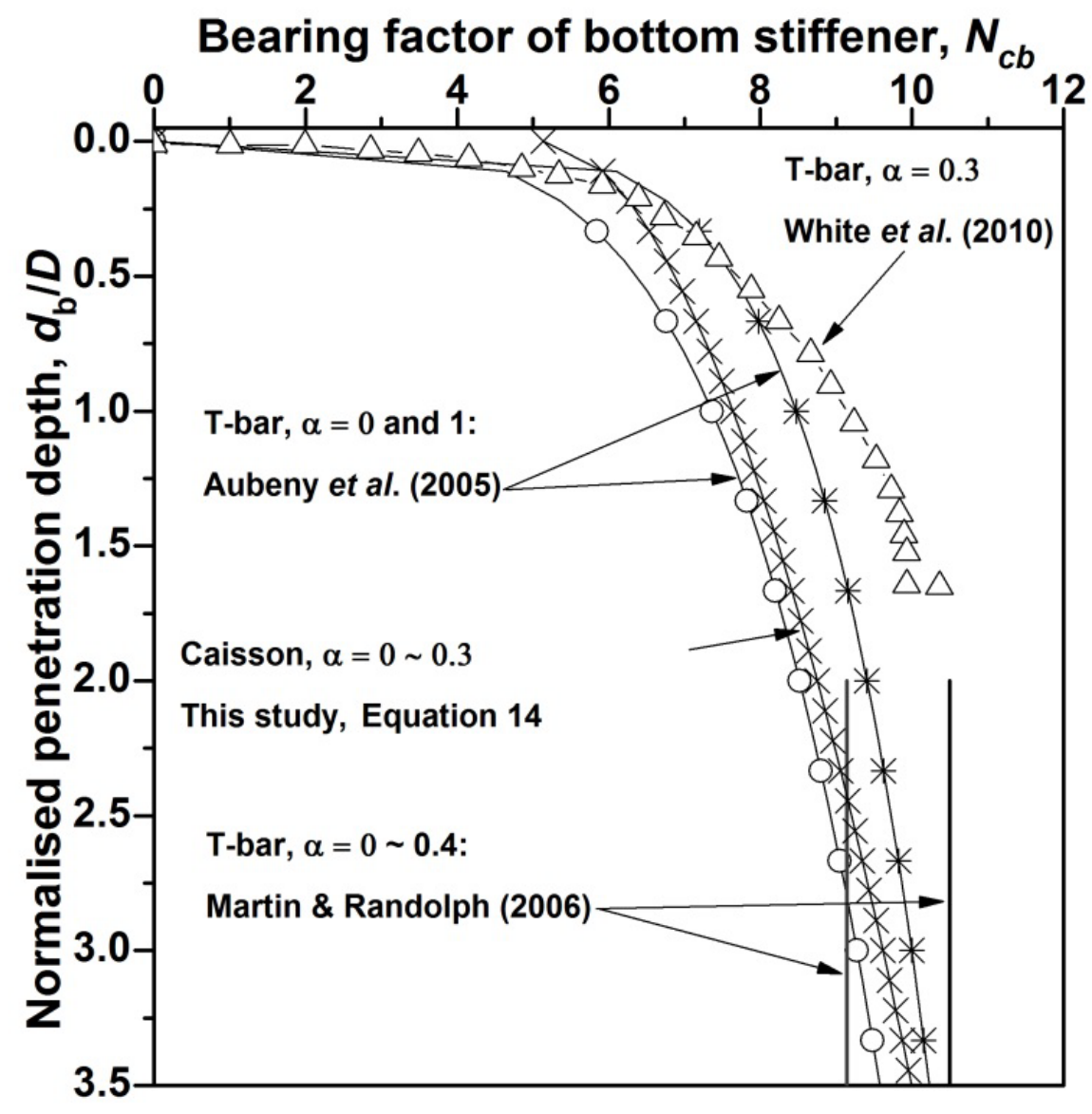

17(c) Proposed design chart for $N_{\mathrm{cb}}$

Figure 17. End bearing factor at bottom stiffener base $\left(\boldsymbol{N}_{\mathrm{cb}}\right)$ 


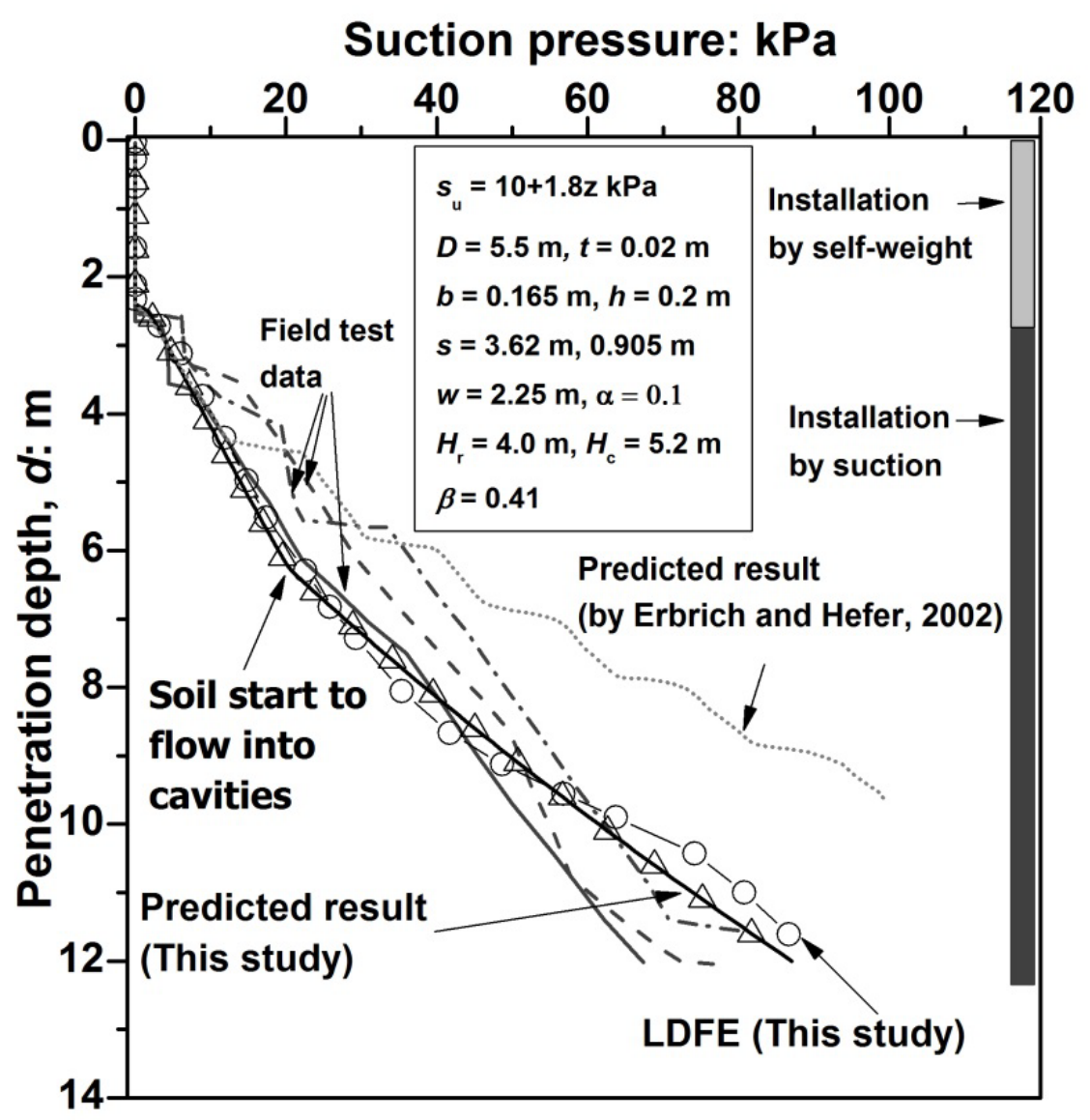

18(a) Comparison with field installation data reported by Erbrich and Hefer (2002) 


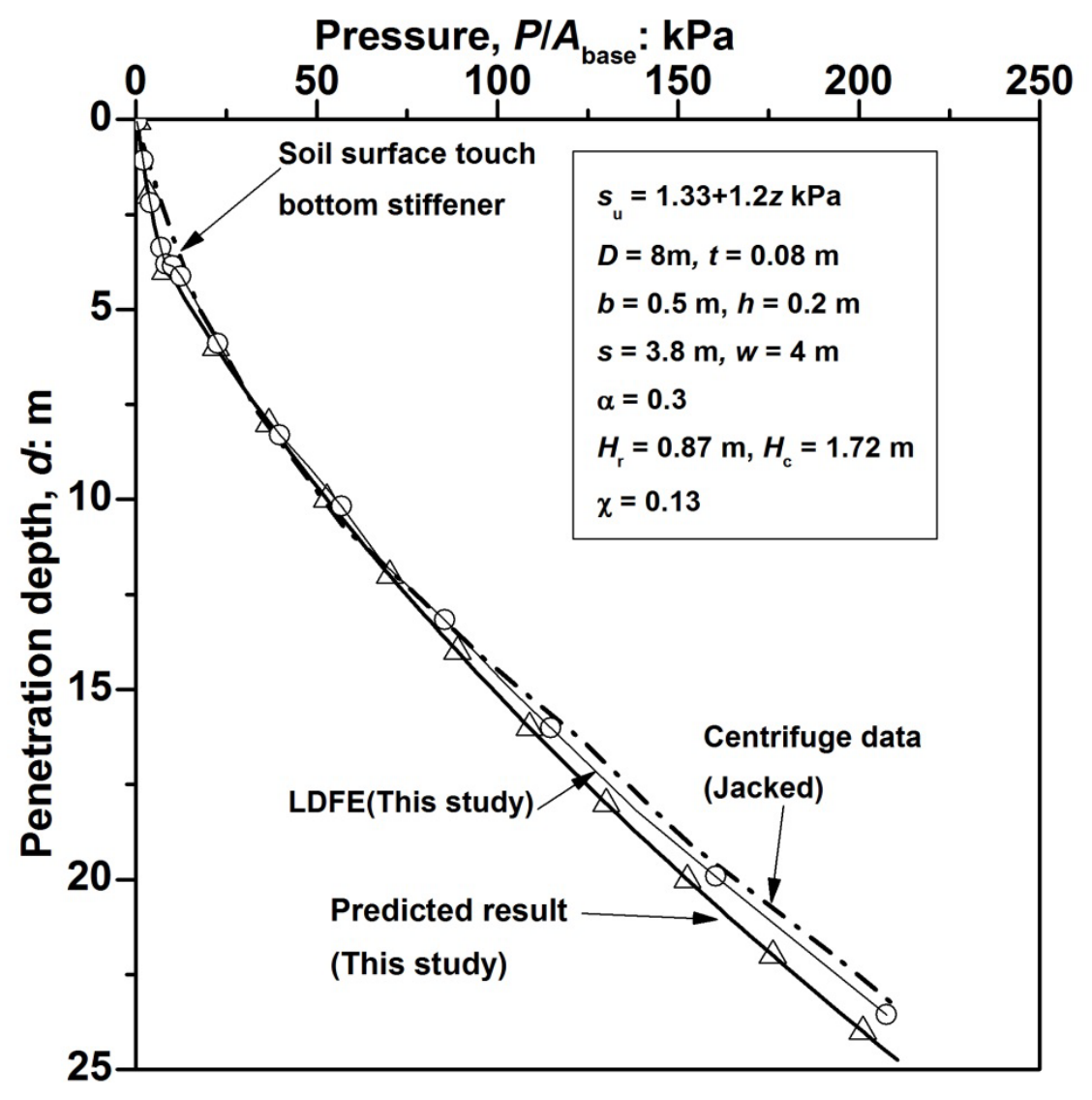

18(b) Comparison with centrifuge test data reported by Gaudin et al. (2014) 


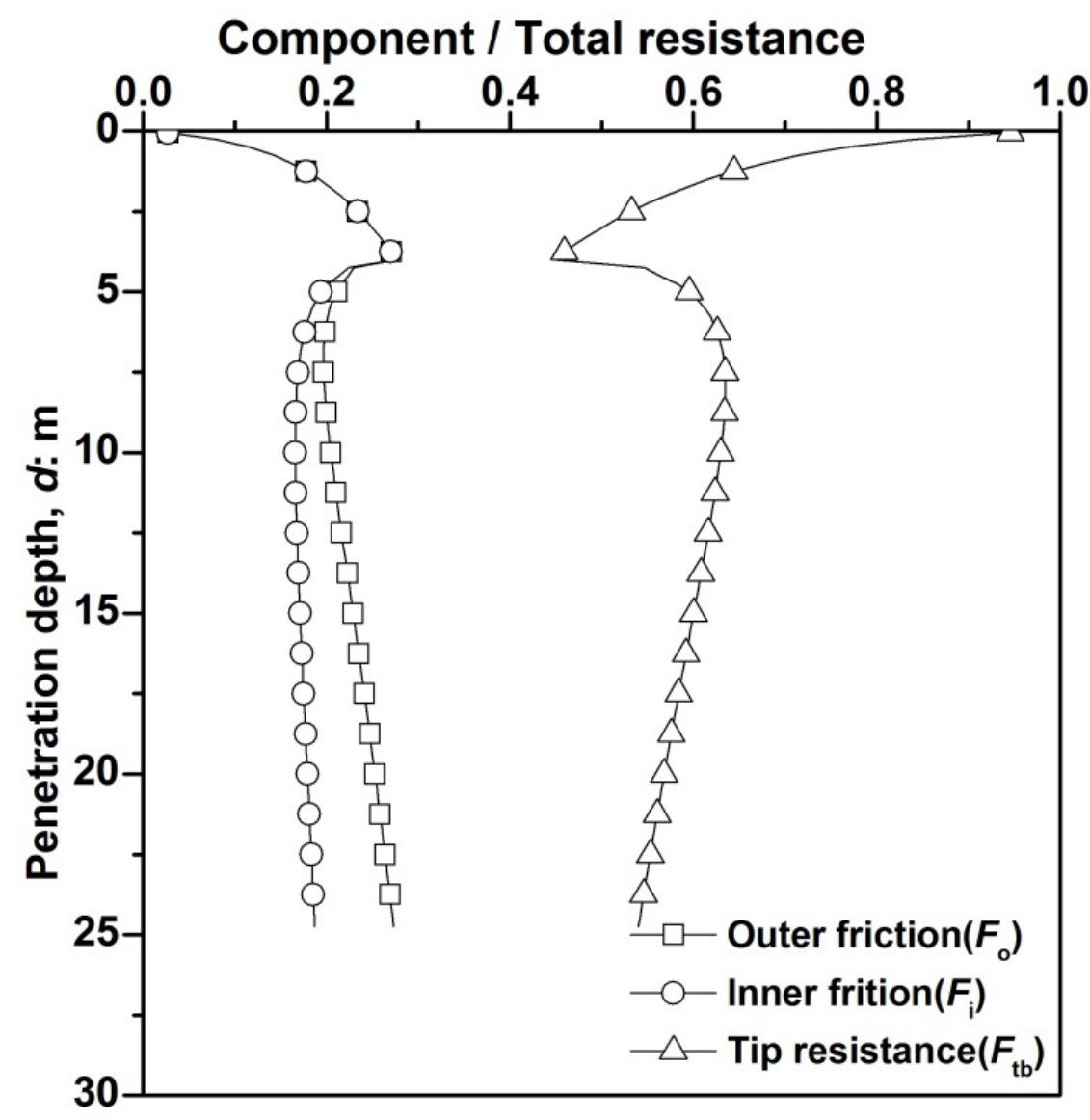

18(c) Sensitivity of different components (stiffened caisson) - predicted results 


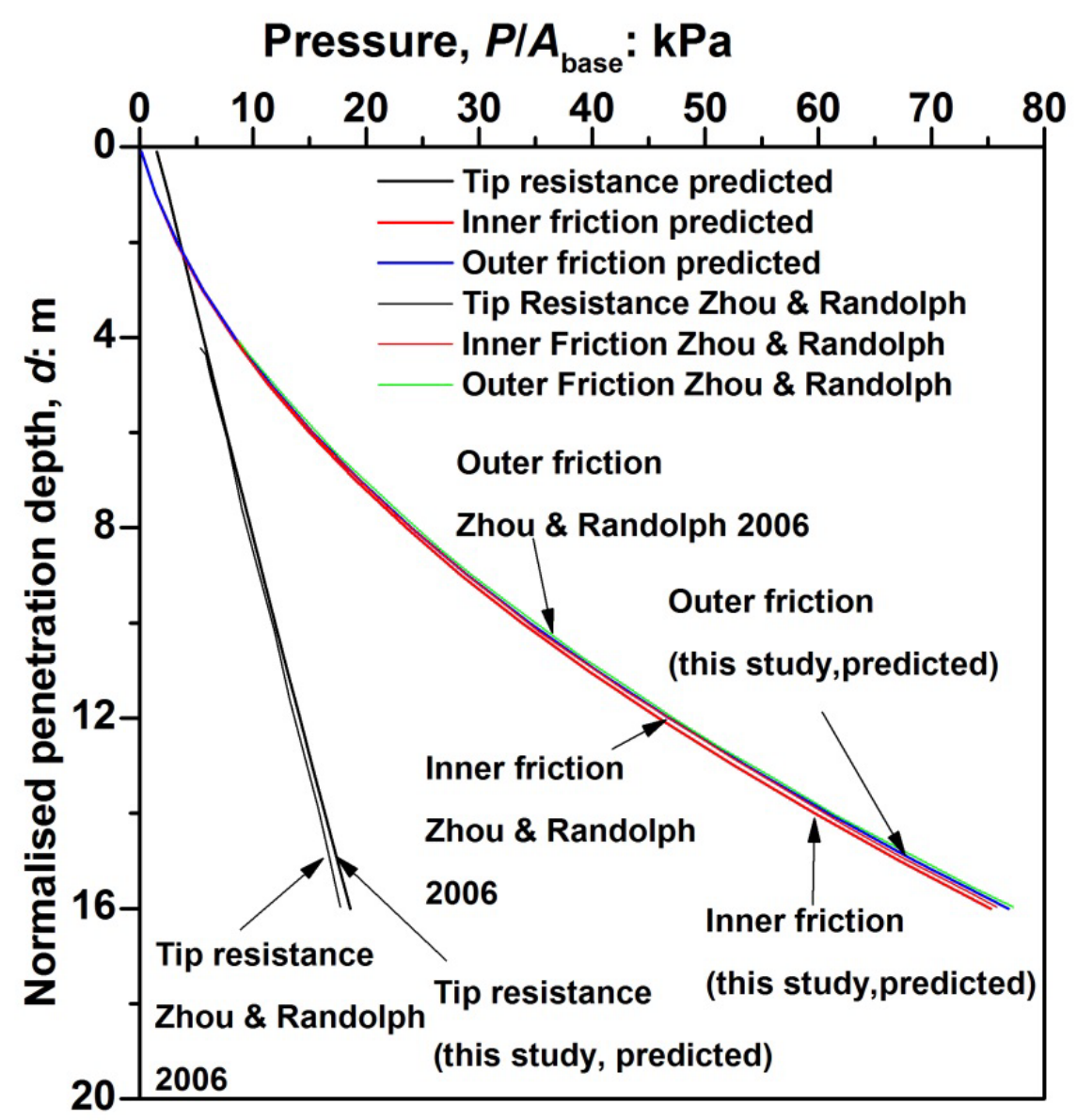

18(d) Sensitivity of different components (unstiffened caisson) - predicted results

Figure 18. Comparison between prediction using proposed mechanism-based design approach and measured and computed data 